\title{
CHARACTERIZATION AND COMPUTATION OF PARTIAL SYNCHRONIZATION MANIFOLDS FOR DIFFUSIVE DELAY-COUPLED SYSTEMS
}

\author{
E. STEUR, H. U. ÜNAL, C. VAN LEEUWEN, AND W. MICHIELS
}

\begin{abstract}
Sometimes a network of dynamical systems shows a form of incomplete synchronization, characterized by synchronization of some but not all of its component systems. This type of incomplete synchronization is called partial synchronization or cluster synchronization. Partial synchronization is associated with the existence of partial synchronization manifolds, which are linear invariant subspaces of $\mathcal{C}$, the state space of the network of systems. We focus on partial synchronization manifolds in networks of identical systems, characterized by linear diffusive coupling described by a weighted graph, and allowing for time-delay in the coupling.

We present equivalent existence criteria for partial synchronization manifolds in terms of invariant spaces, the block structure of a reordered adjacency matrix, and the solvability of a Sylvester equation. We emphasize decomposable networks, according to the rational dependency structure of the coupling weights, and according to the delay values, respectively. It is obvious that if the existence conditions for partial synchronization manifolds are satisfied for all subnetworks simultaneously, they hold for the original network, yet the converse result is not always true, as we shall illustrate with an example. Furthermore, as main results, we show that if the decomposition is according to the weights and the basis weights are rationally independent numbers, or if the decomposition is according to different delay values, then finding a partial synchronization manifold for the original network is equivalent to finding common partial synchronization manifolds for the subnetworks, i.e., restricting to the analysis of the subnetworks does not impose any conservatism, which simplifies the analysis significantly. For the case of decomposable networks according to the weights, with rationally independent basis weights, we provide a fourth existence criterion for partial synchronization manifolds in terms of a balanced coloring of an associated multi-graph. In addition, we briefly describe publicly available software for detecting partial synchronization manifolds.

Our equivalent existence criteria, which depend on the network and delay structure but not on the dynamics of the systems at the nodes, are sufficient for the presence of a partial synchronization manifold. We show that, under a mild assumption on the systems at the nodes, namely left-invertibility, these conditions are necessary as well. In all criteria it turns out that the distinction between non-invasive and invasive delayed coupling is important, i.e., whether or not a coupling term between two systems vanishes whenever the latter are synchronized.
\end{abstract}

\section{INTRODUCTION}

There are many examples of networks of interacting dynamical systems that exhibit collective behavior. Fireflies emit their light pulses at the same instants in time; crickets chirp in unison for extended periods of time; and electrons move 
in synchrony in superconducting Josephson junctions, cf. [40, 30]. The most unambiguous form of collective behavior is that of full synchronization, which refers to the state in which all systems in the network behave identically. However, it is not exceptional that networks do not exhibit completely synchronous behavior but show some kind of incomplete synchronization instead. We study a particular type of this incomplete network synchronization, which we call partial synchronization. Partial synchronization refers to the phenomenon that some systems in the networks are synchronized identically while others are not.

Partial synchronization has been observed, in particular, in biological networks [21]. Central pattern generators produce partially synchronous rhythms that define animal locomotion [10], and synchronization in brains refers in general to the state of coherent activity in parts of the brain [16]. In fact, excessive amounts of synchronization in the brain are a signature of brain disorders like epilepsy and Parkinson's decease [41].

Perhaps surprisingly, partial synchronization does neither require heterogeneous system dynamics nor heterogeneous coupling functions; Partial synchronization is reported in networks of systems which are described by identical vector fields and identical coupling laws $[5,4,32,33]$. In this case the modes of partial synchronization exhibited by the coupled systems are mostly determined by their network structure.

We study how the network structure supports modes of partial synchronization in networks of systems that interact via time-delay linear coupling laws. The networks considered are represented by directed weighted graphs $\mathcal{G}=\mathcal{G}(\mathcal{V}, \mathcal{E}, A)$, where

- $\mathcal{V}$ is a finite set of nodes with cardinality $|\mathcal{V}|=N$;

- $\mathcal{E} \subset \mathcal{V} \times \mathcal{V}$ is the ordered set of edges, where we use the convention that an edge $(i, j) \in \mathcal{E}$ has its head at node $i$ and tail at node $j$;

- $A=\left(a_{i j}\right) \in \mathbb{R}^{N \times N}$ is the weighted adjacency matrix; For an edge $(i, j) \in \mathcal{E}$ constant $a_{i j}>0$ defines the weight of that edge; $a_{i j}=0$ if and only if $(i, j) \notin \mathcal{E}$.

It is clear that all information about the network structure is stored in matrix $A$. hence this matrix will be the central object of our study.

Each node of $\mathcal{G}$ hosts a dynamical system of the form

$$
\left\{\begin{array}{l}
\dot{x}_{i}(t)=f\left(x_{i}(t), u_{i}(t)\right) \\
y_{i}(t)=h\left(x_{i}(t)\right)
\end{array}\right.
$$

where $i \in \mathcal{V}$, state $x_{i}(t) \in \mathbb{R}^{n}$, input(s) $u_{i}(t) \in \mathbb{R}^{m}$, output(s) $y_{i}(t) \in \mathbb{R}^{m}$, function $f: \mathbb{R}^{n} \times \mathbb{R}^{m} \rightarrow \mathbb{R}^{n}$ and function $h: \mathbb{R}^{n} \rightarrow \mathbb{R}^{m}$. The systems (1) on the graph $\mathcal{G}$ interact via either one of the following two types of coupling functions:

$$
u_{i}(t)=\sum_{j \in \mathcal{N}_{i}} a_{i j}\left[y_{j}(t-\tau)-y_{i}(t)\right]
$$

or

$$
u_{i}(t)=\sum_{j \in \mathcal{N}_{i}} a_{i j}\left[y_{j}(t-\tau)-y_{i}(t-\tau)\right] .
$$

Here the set $\mathcal{N}_{i}$ is the neighbor set of node $i \in \mathcal{V}$,

$$
\mathcal{N}_{i}:=\{j \in \mathcal{V} \mid(i, j) \in \mathcal{E}\}
$$

constants $a_{i j}$ are the entries of the weighted adjacency matrix $A$ and constant $\tau$ defines the amount of time-delay. For the time being we focus on the single-delay 
case (in Section 6, we extend our results to the multiple delays case). Functions $f$ and $h$ are assumed to be such that forward solutions of (1), (2) and (1), (3) are uniquely defined on $\left[t_{0}, \infty\right)$, and $h$ is assumed to be injective.

Throughout the paper we will consider only graphs $\mathcal{G}$ that are simple; That is, every pair of nodes of $\mathcal{G}$ is joined by at most one edge and $\mathcal{G}$ does not contain self-loops, which are edges of the form $(i, i), i \in \mathcal{V}$. We remark that the edges of $\mathcal{G}$ model the interaction structure, hence the presence of self-feedback in the coupling functions (2) and (3) (i.e., terms $-\sum_{j \in \mathcal{N}_{i}} a_{i j} y_{i}(t)$ and $-\sum_{j \in \mathcal{N}_{i}} a_{i j} y_{i}(t-$ $\tau)$, respectively) still leaves $G$ simple in our terminology. We remark that in Section 5 , where we discuss decompositions of $\mathcal{G}$, we associate several multi-graphs to the simple graph $\mathcal{G}$. These multi-graphs are allowed to have multiple edges joining the same pair of nodes, but they do not contain self-loops.

In addition, we consider only strongly connected graphs. We recall that a graph is strongly connected if for each pair of nodes $u, v \in \mathcal{V}$ there exists a directed path from $u$ to $v$ and a directed path from $v$ to $u$, cf. [7]. The assumption of a strongly connected graph avoids, e.g., cases in which partial synchronization could occur trivially, such as in two disconnected networks.

The linear coupling functions considered in this paper (possibly with zero delay, i.e. $\tau=0$ ) appear in a large number of applications, such as, networks of electrically coupled neurons $[6,11,22]$, networks of biological systems [29, 35], coupled mechanical systems $[26,34,9,44]$ and electrical systems $[12,43]$. In case of coupling functions (2) the coupling signal $u_{i}(t)$ of node $i$ is defined as the sum of weighted differences of time-delayed outputs of connected systems, $y_{j}(t-\tau)$, and the node's own output at time instant $t, y_{i}(t)$. In this type of coupling the delay models the effect of finite speed of signal transmission. For coupling functions (3) both the node's own output and the outputs of connected systems are delayed by an amount of $\tau$. This type of coupling models the effects of sensor/actuator delay. An example of the latter type of coupling is found in car-following models, where the delay accounts for the human reaction time [36]. We remark that there is a fundamental difference between coupling (2) and coupling (3): The former type of coupling is invasive coupling, which means that the coupling does not vanish when system $i$ and its neighbors are synchronized; The second type of coupling is non-invasive coupling as the coupling inputs $u_{i}(t) \equiv 0$ given that system $i$ is synchronized to its neighbors. This fundamental difference between the two types of coupling functions is reflected in the modes of partial synchronization that a network can exhibit. In particular, as we will show in this paper, modes of partial synchronization that exist in networks of systems with coupling (2) always exist in networks of systems with coupling (3) but the converse is not true. Therefore delay-free coupling, i.e. coupling of the form,

$$
u_{i}(t)=\sum_{j \in \mathcal{N}_{i}} a_{i j}\left[y_{j}(t)-y_{i}(t)\right]
$$

which is clearly non-invasive, should be considered as a special case of coupling (3) rather than coupling (2).

We consider the problem of finding partial synchronization manifolds for networks (represented by a graph $\mathcal{G}$ ) of systems (1) and coupling functions (2) or (3). These partial synchronization manifolds are linear forward invariant manifolds in $\mathcal{C}=\mathcal{C}\left([-\tau, 0], \mathbb{R}^{N n}\right)$, the state-space of the coupled systems, which correspond 
to particular modes of partial synchronization. The identification of partial synchronization manifolds is the first step in the analysis of partial synchronization in networks. The issue of attractivity (or even stability) of partial synchronization manifolds, which is necessary for partial synchronization in any real-world application, is not considered in this paper. For results about stability (and bifurcations) of partial synchronization manifolds we refer to [32, 33, 37, 28].

The "classical" method for identification of partial synchronization manifolds in networks of coupled systems is by looking for symmetries in the dynamical equations of the network, cf. $[13,14,32,15,33,19]$. In particular, the groupoid formalism of [15] is a very useful tool for finding the modes of partial synchronization in a very general class of networks of coupled systems; The systems in that framework do not need to be identical and different types of coupling functions (albeit delay-free coupling) may be present in a network. Algorithms that exploit the groupoid formalism (in combination with lattice theory and tools from linear algebra) for identifying partial synchronization manifolds are presented in, e.g., [1, 2, 20]. Some of our results presented in Sections 3.1, 3.2 and 5 (in particular those involving the invariant subspace condition and its equivalent row-sum condition in case of invasive coupling) may be deducted from the these studies. We also refer to [19] for related results. However, there are important differences between our results and those of $[15,1,2,20]$.

First, our conditions for the existence of partial synchronization manifolds are derived for given systems (1) and given coupling functions (2) or (3) whereas [15, 1, $2,20]$ discuss the existence of partial synchronization manifolds in networks along all admissible vector fields. The restriction to systems of the form (1) and the diffusive time-delay coupling functions (2) or (3) instead of considering all admissible vector fields has two important consequences:

- The conditions for existence of partial synchronization manifolds presented in $[15,1,2,20]$ are necessary and sufficient. To the contrary, our results are sufficient but become necessary only under additional assumptions. See the (counter-)examples in Sections 4 and 5.2.

- In our setting it turns out to be important to make a distinction between invasive coupling and non-invasive coupling; The conditions for existence of partial synchronization manifolds in networks with invasive coupling are more restrictive than those for networks with non-invasive coupling.

For both types of time-delay coupling functions we provide a number of algebraic conditions for the existence of partial synchronization manifolds, namely, an invariant subspace condition, a row-sum condition, and the existence of a solution of a particular matrix equation (which appeared first in [33]). These conditions are shown to be equivalent. In addition we present an algorithm that, for each type of coupling, detects all possible partial synchronization manifolds in a given network. We also identify a class of systems for which our conditions become necessary and we discuss the case of coupling functions with multiple time-delays. The results presented in this paper generalize previous results of the authors $[42,37,38]$. We also generalize the results reported in $[15,20,33,19]$ in the sense that we take timedelays into account and consider diffusive coupling, thereby making (a necessary) distinction between invasive and non-invasive coupling. 
Second, the coupling is described by a weighted adjacency matrix with real valued weights. In this context, another main contribution consists of the characterization of situations where the existence conditions can be checked in exact arithmetic. It follows from our analysis that if the adjacency matrix of a network decomposes according to the interdependency of weights and if the basic weights are rationally independent, then the conditions for existence of partial synchronization manifolds for that network are equivalent to the conditions for existence of partial synchronization manifolds of an associated network described by a multi-graph and corresponding unweighted adjacency matrices. This associated network can be analyzed with tools similar to those in [1], and interpretations will be provided of the conditions for partial synchronization in terms of generalizations of a balanced coloring.

The structure of the paper is as follows. Section 2 is devoted to the problem formulation. Section 3 presents equivalent existence conditions for partial synchronization manifolds of delay-coupled systems, in terms of invariant subspaces of the adjacency matrix or graph Laplacian, in terms of row-sums of the restructured adjacency matrix, and in terms of the solvability of a Sylvester equation. A condition for which the results of Section 3 become necessary is presented in Section 4. Section 5 addresses decomposable graphs based on the rational dependency structure of the weights of their edges. Conditions for the existence of partial synchronization manifolds for coupling functions with multiple time-delays are provided in Section 6. A software implementation of the criteria for partial synchronization manifolds of Sections 3 and 5 is briefly discussed in Section 7. Concluding remarks are provided in Section 8.

\section{Partial SYNChronizATion MANifOLdS}

We consider a given graph $\mathcal{G}=\mathcal{G}(\mathcal{V}, \mathcal{E}, A)$. Without loss of generality we shall assume that

$$
\mathcal{V}=\{1,2, \ldots, N\}
$$

Let $\mathcal{P}$ be a partition of $\mathcal{V}$; That is, $\mathcal{P}$ is a collection of disjoint non-empty subsets of $\mathcal{V}$ with the property that the union of all these subsets equals the set $\mathcal{V}$. These disjoint subsets are referred to as the parts of the partition. One may think of the partitioning of $\mathcal{V}$ as dividing the nodes of the network into clusters, where nodes belonging to the same part of the partition are in the same cluster. The question is now whether the partition is compatible with the dynamics of the systems, coupling functions and interaction structure. If this is the case the network has a partial synchronization manifold and we call that partition a viable partition. We shall parameterize a partial synchronization manifold by a permutation matrix, which corresponds to the partition $\mathcal{P}$ of $\mathcal{V}$. The main motivation for this parametrization by a permutation matrix is that it allows for expressing the conditions for the existence of a partial synchronization manifold algebraically.

In this section we shall first establish a correspondence between a partition $\mathcal{P}$ of the set $\mathcal{V}$ and a permutation matrix. Then we shall introduce the concept of partially synchronous solutions of the coupled systems and introduce the partial synchronization manifolds.

2.1. Characterization of partitions by permutation matrices. Let $\Pi$ be a $N \times N$ permutation matrix. For the network $\mathcal{G}$ we let such a permutation matrix 
$\Pi$ define a partition $\mathcal{P}$ of the set of nodes $\mathcal{V}$ as follows. Given two nodes $i, j \in \mathcal{V}$, we let $i \sim j$, with $\sim$ denoting an equivalence relation, if the $i j^{\text {th }}$ entry of $\Pi$ is equal to 1 . Note that, because of the transitivity of the equivalence relation, we have that $\Pi_{i j}=1$ and $\Pi_{j \ell}=1$ implies $i \sim \ell$. The set of equivalence classes then defines a partition $\mathcal{P}$ of $\mathcal{V}$. (Recall that the equivalence class of $i$ in $\mathcal{V}$ is the set $\{j \in \mathcal{V} \mid i \sim j\})$. Then a part of the partition $\mathcal{P}$ is a subset of $\mathcal{V}$ containing all nodes that belong to the same equivalence class. It can easily be shown that the number of parts is equal to

$$
K:=\operatorname{dim} \operatorname{ker}\left(I_{N}-\Pi\right),
$$

where $I_{N}$ is the $N \times N$ identity matrix. We let $k_{\ell}$ denote the size of the $\ell^{\text {th }}$ part of $\mathcal{P}$, where $\ell=1,2, \ldots, K$.

Conversely, to a given partition $\mathcal{P}$ of $\mathcal{V}$ with $K$ parts we associate a $N \times N$ permutation matrix $\Pi$ with $\operatorname{dim} \operatorname{ker}\left(I_{N}-\Pi\right)=K$ as follows. Let $\mathcal{P}=\left\{\mathcal{P}_{1}, \ldots, \mathcal{P}_{N}\right\}$ be given, where $\mathcal{P}_{\ell}$ is a part of the partition, $\ell=1, \ldots, K$, and let $k_{\ell}$ be the size of $\mathcal{P}_{\ell}$. If $\mathcal{P}_{\ell}=\{i\}$, i.e. $k_{\ell}=1$, then set the $i i^{\text {th }}$ entry of $\Pi$ equal to one. If $\mathcal{P}_{\ell}=\left\{j_{1}, j_{2}, \ldots, j_{k_{\ell}}\right\}$ then set the $j_{1} j_{2}^{\text {th }}$ entry of $\Pi$ equal to one, $\ldots$, and finally the $j_{k_{\ell}} j_{1}^{\text {th }}$ entry of $\Pi$ equal to one. Thus for example, if $\mathcal{V}=\{1,2,3,4,5\}$ and $\mathcal{P}=\left\{\mathcal{P}_{1}, \mathcal{P}_{2}\right\}=\{\{1,3,4\},\{2,5\}\}$, then the construction above gives

$$
\Pi=\left(\begin{array}{lllll}
0 & 0 & 1 & 0 & 0 \\
0 & 0 & 0 & 0 & 1 \\
0 & 0 & 0 & 1 & 0 \\
1 & 0 & 0 & 0 & 0 \\
0 & 1 & 0 & 0 & 0
\end{array}\right) .
$$

We remark that a permutation matrix defines a partition $\mathcal{P}$ of $\mathcal{V}$ uniquely but the converse is not necessarily true. Indeed, the permutation matrix

$$
\Pi=\left(\begin{array}{lllll}
0 & 0 & 0 & 1 & 0 \\
0 & 0 & 0 & 0 & 1 \\
1 & 0 & 0 & 0 & 0 \\
0 & 0 & 1 & 0 & 0 \\
0 & 1 & 0 & 0 & 0
\end{array}\right)
$$

defines the same partition $\mathcal{P}=\{\{1,3,4\},\{2,5\}\}$ of $\mathcal{V}=\{1,2,3,4,5\}$.

2.2. Definition of partial synchronization manifolds. Consider the coupled systems (1), (2) or (1), (3) on a given graph $\mathcal{G}$. Let $\mathcal{C}\left([-\tau, 0], \mathbb{R}^{N n}\right)$ be the space of continuous functions that map the interval $[-\tau, 0] \subset \mathbb{R}$ into $\mathbb{R}^{N n}$. Then given initial data $\psi \in \mathcal{C}\left([-\tau, 0], \mathbb{R}^{N n}\right)$ we denote by $x_{t} \in \mathcal{C}\left([-\tau, 0], \mathbb{R}^{N n}\right)$, where

$$
x_{t}(\theta):=\left(\begin{array}{c}
x_{t, 1}(\theta) \\
x_{t, 2}(\theta) \\
\vdots \\
x_{t, N}(\theta)
\end{array}\right)=\left(\begin{array}{c}
x_{1}(t+\theta) \\
x_{2}(t+\theta) \\
\vdots \\
x_{N}(t+\theta)
\end{array}\right), \quad \theta \in[-\tau, 0],
$$

a segment of a solution of the coupled systems (1), (2) or (1), (3) that coincides with $\psi$ at $t=t_{0}$, i.e. $x_{t_{0}}=\psi$. Such a solution is a partially synchronous solution if there exist $i, j \in \mathcal{V}$ with $i \neq j$ such that

$$
x_{t, i}=x_{t, j}, \quad \forall t \geq t_{0} .
$$

Of course, if the above equality holds for all $i, j \in \mathcal{V}$ we have full synchronization. 
By means of an $N \times N$ permutation matrix $\Pi$ we can write conditions (4) in a more compact form:

$$
x_{t}(\theta)=\left(\Pi \otimes I_{n}\right) x_{t}(\theta), \quad \forall \theta \in[-\tau, 0], \quad \forall t \geq t_{0},
$$

where $\otimes$ denotes the Kronecker product (tensor product) of two matrices. Note that we can rewrite (5) as

$$
x_{t}(\theta) \in \operatorname{ker}\left(I_{N n}-\Pi \otimes I_{n}\right), \quad \forall \theta \in[-\tau, 0], \quad \forall t \geq t_{0},
$$

or as $x_{t} \in \mathcal{M}(\Pi), \forall t \geq t_{0}$, where

$$
\begin{aligned}
\mathcal{M}(\Pi):=\left\{\phi \in \mathcal{C}\left([-\tau, 0], \mathbb{R}^{N n}\right) \mid \phi(\theta)=\operatorname{col}\left(\phi_{1}(\theta), \phi_{2}(\theta), \ldots, \phi_{N}(\theta)\right),\right. & \\
& \left.\phi_{i}(\theta) \in \mathbb{R}^{n}, i=1, \ldots, N, \phi(\theta) \in \operatorname{ker}\left(I_{N n}-\Pi \otimes I_{n}\right) \forall \theta \in[-\tau, 0]\right\}
\end{aligned}
$$

is the set of partially synchronous states induced by the permutation matrix $\Pi$ (i.e., for which the synchronized clusters are defined by the partition induced by $\Pi$ ).

It is now clear that for the existence of a partially synchronous solution we require

$$
1<K=\operatorname{dim} \operatorname{ker}\left(I_{N}-\Pi\right)<N,
$$

where $K$ denotes the number of synchronized clusters. Here a synchronized cluster, or simply, a cluster, refers to the subset of nodes with synchronized dynamics. These clusters are exactly parts of the partition $\mathcal{P}$ of $\mathcal{V}$ associated with $\Pi$. Thus for $K=1$ we have full synchronization, i.e.

$$
x_{t, i}=x_{t, j}, \quad \forall i, j \in \mathcal{V}, \quad \forall t \geq t_{0},
$$

and $K=N$ corresponds to the case of no synchronization. Note that $K=N$ if and only if $\Pi=I_{N}$. We now define a partial synchronization manifold as follows:

Definition 1. The set $\mathcal{M}(\Pi)$ with permutation matrix $\Pi$ for which $1<K<N$ is a partial synchronization manifold for the coupled systems (1), (2), or (1), (3), if and only if it is positively invariant under the dynamics (1), (2), or (1), (3), respectively.

With the terminology introduced above and the fact that our systems (1) are assumed to be identical, it is clear that $\mathcal{M}(\Pi)$ is a partial synchronization manifold for the coupled systems (1), (2) or (1), (3) if systems that belong to the same cluster receive identical inputs for all $t \geq t_{0}$ :

$$
x_{t}(\theta)=\left(\Pi \otimes I_{N}\right) x_{t}(\theta) \Rightarrow y_{t}(\theta)=\left(\Pi \otimes I_{m}\right) y_{t}(\theta) \Rightarrow u(t)=\left(\Pi \otimes I_{m}\right) u(t),
$$

with $-\tau \leq \theta \leq 0$ and

$$
y_{t}(\theta)=\left(\begin{array}{c}
y_{1}(t+\theta) \\
\vdots \\
y_{N}(t+\theta)
\end{array}\right), \quad u(t)=\left(\begin{array}{c}
u_{1}(t) \\
\vdots \\
u_{N}(t)
\end{array}\right) .
$$

Note that $x_{t}(\theta)=\left(\Pi \otimes I_{N}\right) x_{t}(\theta) \Rightarrow y_{t}(\theta)=\left(\Pi \otimes I_{m}\right) y_{t}(\theta)$ follows from the assumption that the output map $h$ is injective. As we discuss in Section 4, the condition of equal inputs to systems in the same cluster, which is sufficient for $\mathcal{M}(\Pi)$ to be a partial synchronization manifold, is close to necessary. Because

$$
u(t)=\left(A \otimes I_{m}\right) y(t-\tau)-\left(D \otimes I_{m}\right) y(t)
$$

for coupling functions $(2)$, and

$$
u(t)=\left((A-D) \otimes I_{m}\right) y(t-\tau)=-\left(L \otimes I_{m}\right) y(t-\tau)
$$


for coupling functions (3), where

$$
D=\left(\begin{array}{ccc}
\sum_{j=2}^{N} a_{1 j} & & 0 \\
& \ddots & \\
0 & & \sum_{j=1}^{N-1} a_{N j}
\end{array}\right)
$$

and $L:=D-A$ is the weighted Laplacian matrix, it follows readily that the conditions for the existence of partial synchronization manifolds for a given network of systems (1), (2) or (1), (3) can be deducted from the matrix $A$ (or its associated Laplacian matrix $L)$.

\section{Algebraic CONDitions For the EXistence of PARTial SyNCHROnization MANIFOLDS}

In this section we provide for a given network with adjacency matrix $A$ and given permutation matrix $\Pi$ for each types of coupling three equivalent algebraic conditions for $\mathcal{M}(\Pi)$ to be a partial synchronization manifold.

3.1. Invariant subspaces of $A$ and $L$. Our first set of conditions for the existence of a partial synchronization manifold expresses the implication

$$
y_{t}(\theta)=\left(\Pi \otimes I_{m}\right) y_{t}(\theta) \Rightarrow u(t)=\left(\Pi \otimes I_{m}\right) u(t), \quad-\tau \leq \theta \leq 0,
$$

explicitly in terms of the matrices $A$ (for coupling (2)) and $L$ (for coupling (3)). Recall that the statement above simply means that systems that belong to the same cluster receive identical inputs.

Proposition 1. Consider the coupled systems (1), (2) on a graph $\mathcal{G}=(\mathcal{V}, \mathcal{E}, A)$ and consider a $N \times N$ permutation matrix $\Pi$. If $\operatorname{ker}\left(I_{N}-\Pi\right)$ is a right invariant subspace of $A$, i.e. $A V \subset V$ where $V:=\operatorname{ker}\left(I_{N}-\Pi\right)$, then $\mathcal{M}(\Pi)$ is a partial synchronization manifold for the coupled systems (1), (2).

Proof. Observe that $\mathbf{1}_{N} \in \operatorname{ker}\left(I_{N}-\Pi\right)$, where $\mathbf{1}_{N}$ is the $N$-dimensional vector with all entries equal to 1 . Because $A \mathbf{1}_{N}=D \mathbf{1}_{N} \in \operatorname{ker}\left(I_{N}-\Pi\right)$ we conclude that $\operatorname{ker}\left(I_{N}-\Pi\right)$ being an right invariant subspace of $A$ implies that $\operatorname{ker}\left(I_{N}-\Pi\right)$ is a right invariant subspace of $D$. For coupled systems (1), (2), for any $y_{t}(\theta) \in$ $\operatorname{ker}\left(I_{N m}-\Pi \otimes I_{m}\right)$, which is equivalent to $y_{t}(\theta)=\left(\Pi \otimes I_{m}\right) y_{t}(\theta)$, the invariant subspace condition implies

$$
u(t)=-\left(D \otimes I_{m}\right) y(t)+\left(A \otimes I_{m}\right) y(t-\tau) \in \operatorname{ker}\left(I_{N m}-\Pi \otimes I_{m}\right),
$$

i.e. $u(t)=\left(\Pi \otimes I_{m}\right) u(t)$.

Proposition 2. Consider the coupled systems (1), (3) on a graph $\mathcal{G}=(\mathcal{V}, \mathcal{E}, A)$ and consider a $N \times N$ permutation matrix $\Pi$. If $\operatorname{ker}\left(I_{N}-\Pi\right)$ is a right invariant subspace of $L=D-A$, then $\mathcal{M}(\Pi)$ is a partial synchronization manifold for the coupled systems (1), (3).

Proof. Analogous to the proof of Proposition 1.

Remark 1. As $\operatorname{ker}\left(I_{N}-\Pi\right)$ being a right invariant subspace of $A$ implies $\operatorname{ker}\left(I_{N}-\Pi\right)$ being a right invariant subspace of $D$, it follows that $\operatorname{ker}\left(I_{N}-\Pi\right)$ is also a right invariant subspace of $L=D-A$. Thus the condition of Proposition 1 is also sufficient for $\mathcal{M}(\Pi)$ to be a partial synchronization manifold for coupled systems (1), (3). However, the converse is in general not true. 
Propositions 1 and 2 allow us to determine whether a permutation matrix corresponds to a partial synchronization manifold by identifying repeating patterns in the (generalized) eigenvectors of matrices $A$ and $L$, respectively. An example is provided at the end of this section. However, we remark that the computation of generalized eigenvectors has a computational cost of $\mathcal{O}\left(N^{3}\right)$ and is prone to numerical errors.

The invariant subspace condition for coupling functions (2) was introduced first in [38]. However, in that paper the authors made the additional assumption that $\operatorname{ker}\left(I_{n}-\Pi\right)$ is a right invariant subspace of $D$. Here this additional assumption is redundant. The invariant subspace condition for coupling functions (3) was introduced first in [42] for a more restrictive class of systems.

3.2. Block-structured adjacency matrix. For our second set of conditions we relabel the nodes of our network such that the first $k_{1}$ nodes belong to cluster 1 , the second $k_{2}$ nodes belong to cluster 2, and so on. Mathematically this relabeling can be done using a permutation matrix, which we denote by $R$ and we shall refer to this matrix $R$ as the reordering matrix. For a given $N \times N$ permutation matrix $\Pi$ with $1<K=\operatorname{dim} \operatorname{ker}\left(I_{N}-\Pi\right)<N$, it is always possible to find an $N \times N$ reordering matrix $R$ such that

$$
R^{\top} \Pi R=\bar{\Pi}=\bar{\Pi}\left(k_{1}, \ldots, k_{K}\right):=\left(\begin{array}{llll}
\Pi_{C}\left(k_{1}\right) & & & \\
& \Pi_{C}\left(k_{2}\right) & & \\
& & \ddots & \\
& & & \Pi_{C}\left(k_{K}\right)
\end{array}\right),
$$

i.e. $\bar{\Pi}$ is a block diagonal matrix with $K$ blocks $\Pi_{C}\left(k_{\ell}\right)$, each of which is a $k_{\ell} \times k_{\ell^{-}}$ dimensional cyclic permutation matrix

$$
\Pi_{C}\left(k_{\ell}\right)=\left(\begin{array}{ccccc}
0 & 0 & \cdots & 0 & 1 \\
1 & 0 & \cdots & 0 & 0 \\
0 & \ddots & \ddots & \vdots & \vdots \\
\vdots & \ddots & \ddots & 0 & 0 \\
0 & \cdots & 0 & 1 & 0
\end{array}\right) \in \mathbb{R}^{k_{\ell} \times k_{\ell}},
$$

and $\Pi_{C}(1)=1$. The reordering matrix $R$ can be easily constructed using the following procedure. First determine the partition $\mathcal{P}$ that corresponds to $\Pi$ (See Section 2.1). Then relabel the nodes such that the first $k_{1}$ nodes belong to part 1 of $\mathcal{P}$, the next $k_{2}$ nodes belong to part 2 of $\mathcal{P}$, and so on. Then $R$ is the matrix that defines to this relabeling procedure. Given matrices $\Pi$ and $A$, let the reordering matrix $R$ be such that $R^{\top} \Pi R$ is of the form (6), and determine the reordered adjacency matrix

$$
R^{\top} A R=\left(\begin{array}{c:c:c:c}
A_{11} & A_{12} & \cdots & A_{1 K} \\
\hdashline A_{21} & \bar{A}_{22} & \cdots & A_{2 K}^{-} \\
\hdashline \vdots & \ddots & \ddots & \vdots \\
\hdashline A_{K 1} & \bar{A}_{K 2} & \cdots & \bar{A}_{K K}
\end{array}\right), \quad A_{i j} \in \mathbb{R}^{k_{i} \times k_{j}}
$$

Note that the block structure in the reordered adjacency matrix (8) is completely determined by $\Pi$ and $R$. 
Lemma 1. Given an adjacency matrix $A$ and a permutation matrix $\Pi$ of the same dimension. Associate with $\Pi$ a reordering matrix $R$ such that $R^{\top} \Pi R$ is of the form (6). The following statements are equivalent:

1) $\operatorname{ker}\left(I_{N}-\Pi\right)$ is a right invariant subspace of $A$;

2) all blocks of the block-structured reordered adjacency matrix (8) have constant row-sums.

Proof. $2 \Rightarrow 1$. Without loss of generality we assume that $\Pi$ takes the form (6) and $A$ has block-structure (8). Then $R=I_{N}$ and any vector $v \in \operatorname{ker}\left(I_{N}-\Pi\right)$ is of the form

$$
v=\nu_{1} e_{1}+\nu_{2} e_{2}+\cdots+\nu_{K} e_{K}
$$

where $\nu_{1}, \ldots, \nu_{K}$ are constants and

$$
e_{1}=\left(\begin{array}{c}
\mathbf{1}_{k_{1}} \\
\mathbf{0}_{k_{2}} \\
\vdots \\
\mathbf{0}_{k_{K}}
\end{array}\right), \quad e_{2}=\left(\begin{array}{c}
\mathbf{0}_{k_{1}} \\
\mathbf{1}_{k_{2}} \\
\vdots \\
\mathbf{0}_{k_{K}}
\end{array}\right), \quad \ldots, \quad e_{K}=\left(\begin{array}{c}
\mathbf{0}_{k_{1}} \\
\vdots \\
\mathbf{0}_{k_{K-1}} \\
\mathbf{1}_{k_{K}}
\end{array}\right),
$$

where $k_{\ell}=\operatorname{dim}\left(A_{\ell \ell}\right)$ and $\mathbf{0}_{q}$ denotes the $q$-dimensional vector with all entries equal to 0 . Clearly

$$
A v=\nu_{1}\left(\begin{array}{c}
A_{11} \mathbf{1}_{k_{1}} \\
A_{21} \mathbf{1}_{k_{1}} \\
\vdots \\
A_{K 1} \mathbf{1}_{k_{1}}
\end{array}\right)+\nu_{2}\left(\begin{array}{c}
A_{12} \mathbf{1}_{k_{2}} \\
A_{22} \mathbf{1}_{k_{2}} \\
\vdots \\
A_{K 2} \mathbf{1}_{k_{2}}
\end{array}\right)+\cdots+\nu_{K}\left(\begin{array}{c}
A_{1 K} \mathbf{1}_{k_{K}} \\
A_{2 K} \mathbf{1}_{k_{K}} \\
\vdots \\
A_{K K} \mathbf{1}_{k_{K}}
\end{array}\right)
$$

If condition 2 holds, then

$$
A v=\nu_{1}\left(\rho_{11}+\rho_{21}+\cdots+\rho_{K 1}\right) e_{1}+\cdots+\nu_{K}\left(\rho_{1 K}+\rho_{2 K}+\cdots+\rho_{K K}\right) e_{K},
$$

where $\rho_{i j} \mathbf{1}_{k_{j}}=A_{i j} \mathbf{1}_{k_{j}}$. Thus $A v \in \operatorname{ker}\left(I_{N}-\Pi\right)$ for any $v \in \operatorname{ker}\left(I_{N}-\Pi\right)$.

$1 \Rightarrow 2$. Let again, without loss of generality, $\Pi$ being of the form (6) and $A$ has block-structure (8) such that $R=I_{N}$. Condition 1 states that for any vector

$$
v=\nu_{1} e_{1}+\nu_{2} e_{2}+\cdots+\nu_{K} e_{K}
$$

where vectors $e_{\ell}$ are as defined above and $\nu_{\ell}$ are arbitrary constants,

$$
A v=\left(\begin{array}{c}
\sum_{j=1}^{K} \nu_{j} A_{1 j} \mathbf{1}_{k_{j}} \\
\mathbf{0}_{k_{2}} \\
\vdots \\
\mathbf{0}_{k_{K}}
\end{array}\right)+\cdots+\left(\begin{array}{c}
\mathbf{0}_{k_{1}} \\
\vdots \\
\mathbf{0}_{k_{K-1}} \\
\sum_{j=1}^{K} \nu_{j} A_{K j} \mathbf{1}_{k_{j}}
\end{array}\right) \in \operatorname{ker}\left(I_{N}-\Pi\right) .
$$

Take $\nu_{1} \neq 0$ and all other $\nu_{\ell}=0$. Then the equation

$$
A v=\nu_{1}\left(\begin{array}{c}
A_{11} \mathbf{1}_{k_{1}} \\
A_{21} \mathbf{1}_{k_{2}} \\
\vdots \\
A_{K 1} \mathbf{1}_{k_{K}}
\end{array}\right) \in \operatorname{ker}\left(I_{N}-\Pi\right)
$$

requires that all blocks $A_{\ell 1}, \ell=1, \ldots, K$, to have constant row-sums. By repetition of this argument (i.e. considering all vectors $v \in \operatorname{ker}\left(I_{k}-\Pi\right)$ of the form $v=\nu_{\ell} e_{\ell}$ with non-zero $\nu_{\ell}$ ) we conclude that condition 1 implies that all block $A_{i j}$ have constant row-sums. 
Recall that, by Proposition 1, if the conditions of the lemma are satisfied then $\mathcal{M}(\Pi)$ is a partial synchronization manifold for the coupled systems (1), (2). In fact, $\mathcal{M}(\Pi)$ is also a partial synchronization manifold for coupled systems (1), (3). A similar result was obtained in [20, 27]. Unlike these studies, we consider delayed diffusive coupling type, where the distinction between invasive coupling and non-invasive coupling is crucial. Namely, as we show next, the constant rowsum condition on the blocks of the adjacency matrix can be relaxed in case of non-invasive coupling (3).

Lemma 2. Given an adjacency matrix $A$ and a permutation matrix $\Pi$ of the same dimension. Associate with $\Pi$ a reordering matrix $R$ such that $R^{\top} \Pi R$ is of the form (6). The following statements are equivalent:

1) $\operatorname{ker}\left(I_{N}-\Pi\right)$ is a right invariant subspace of the Laplacian matrix $L=D-A$ associated to $A$;

2) all off-diagonal blocks of the block-structured reordered adjacency matrix (8) have constant row-sums.

Proof. Let, without loss of generality, $\Pi$ have the form (6) and $A$ have blockstructure (8) such that $R=I_{N}$. Assign matrix $D$ the same block-structure as the (reordered) matrix $A$,

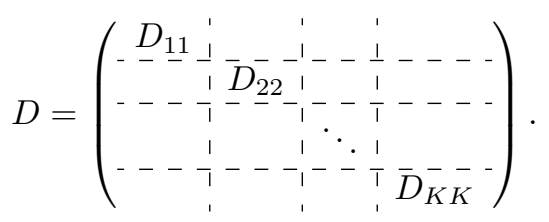

Hence the matrix $L$ has the same block-structure as $A$. By construction, $L \mathbf{1}_{N}=\mathbf{0}_{N}$, with $\mathbf{0}_{N}$ the $N$-dimensional vector of zeros. Then the condition that all off-diagonal blocks of the matrix $A$ having constant row-sums is equivalent to all blocks of $L$ having constant row-sums. Using this fact the proof of the lemma is obtained from the proof of Lemma 1 when replacing $A$ by $L$.

By Proposition 2, if the conditions above hold true, then $\mathcal{M}(\Pi)$ is a partial synchronization manifold for the coupled systems (1), (3).

An advantage of the row-sum test of the block-structured adjacency matrix over an eigenvalue decomposition is that the computational cost is $\mathcal{O}\left(N^{2}\right)$ (provided that $A$ has been reordered). Moreover, as we show in Section 7, in many practical cases the row-sum condition can be verified in exact arithmetic.

3.3. A Sylvester equation. Our third set of algebraic conditions for $\Pi$ to define a partial synchronization manifold involves the existence of a solution to a Sylvester equation. This particular equation was introduced in [33], extending the results of [32], in a study of partial synchronization in networks of systems with delay-free coupling of the form

$$
u_{i}(t)=\sum_{j \in \mathcal{N}_{i}} a_{i j}\left[y_{j}(t)-y_{i}(t)\right],
$$

where $(i, j) \in \mathcal{E} \Leftrightarrow(j, i) \in \mathcal{E}$ and $a_{i j}=a_{j i}$. It was shown that $\mathcal{M}(\Pi)$ is a partial synchronization manifold if the Sylvester equation

$$
\left(I_{N}-\Pi\right) L=X\left(I_{N}-\Pi\right)
$$


has a solution $X \in \mathbb{R}^{N \times N}$. In the particular case that $\Pi$ and $L$ commute, i.e. $\Pi L=L \Pi$, the equation has a solution $X=L$, cf. [32]. The condition of commuting $\Pi$ and $L$ (or $\Pi$ and $A$ in case of invasive coupling) is often used in the study of partial synchronization in networks, cf. [24, 19, 28]. We wish to emphasize that commuting $\Pi$ and $L$ (or $A$ ) is sufficient for the existence of a partial synchronization manifold but not necessary. An example of a network for which $\Pi$ and $L$ do not commute but there exist a partial synchronization manifold is found, for instance, in $[33]$.

The matrix $X$ plays an important role in the stability analysis of partial synchronization; For a particular class of systems one can show that the partial synchronization manifold $\mathcal{M}(\Pi)$ contains an asymptotically stable subset if there is a positive number $\gamma$, which satisfies

$$
\left(I_{N}-\Pi\right)^{\top}\left(X^{\top}+X\right)\left(I_{N}-\Pi\right) \geq \gamma\left(I_{N}-\Pi\right)^{\top}\left(I_{N}-\Pi\right)
$$

and this number exceeds some critical threshold value (that depends on the vector field of the systems), cf. [33].

The results of [33] were extended in [37] to the case of asymmetric adjacency matrices and directed graphs, and time-delay coupling functions (2) or (3). Extended conditions for the existence of partial synchronization manifolds in such networks of time-delay coupled systems are presented below; We refer to [37] for related results on the stability of a partial synchronization manifold. We remark that the stability conditions depend on the matrices $X_{A}$ and $X_{L}$ defined in the two lemmas below. A proof of these two lemmas is found in $[38,42]$. We do present a proof of the lemmas in this manuscript as it is instructive for the construction of the matrices $X_{A}$ and $X_{L}$.

Lemma 3. Given an adjacency matrix $A$ and a permutation matrix $\Pi$, the following statements are equivalent:

1) the set $\operatorname{ker}\left(I_{N}-\Pi\right)$ is a right invariant subspace of $A$;

2) there exists a solution $X_{A} \in \mathbb{R}^{N \times N}$ to the matrix equation

$$
\left(I_{N}-\Pi\right) A=X_{A}\left(I_{N}-\Pi\right)
$$

Proof. $1 \Rightarrow 2$. Consider the singular value decomposition of $I_{N}-\Pi$,

$$
I_{N}-\Pi=V \Sigma W^{\top}
$$

where, without loss of generality,

$$
V=\left(\begin{array}{ll}
V_{1} & V_{2}
\end{array}\right), \quad \Sigma=\left(\begin{array}{cc}
\Sigma_{1} & \\
& 0_{K \times K}
\end{array}\right), \quad W=\left(\begin{array}{ll}
W_{1} & W_{2}
\end{array}\right),
$$

with $V_{1}, W_{1} \in \mathbb{R}^{N \times(N-K)}, V_{2}, W_{2} \in \mathbb{R}^{N \times K}$ and $\Sigma_{1} \in \mathbb{R}^{(N-K) \times(N-K)}$. Note that $\operatorname{ker}\left(I_{N}-\Pi\right)=\operatorname{span}\left(W_{2}\right)$. Given that condition 1 holds, we consider the equation

$$
\left(I_{N}-\Pi\right) A W=X_{A} V \Sigma
$$

and we conclude that

$$
\left(I_{N}-\Pi\right) A W_{2}=0 .
$$

Let $Z=X_{A} V$,

$$
Z=\operatorname{col}\left(\frac{1}{\sigma_{1}}\left(I_{N}-\Pi\right) A w_{1}, \ldots, \frac{1}{\sigma_{N-K}}\left(I_{N}-\Pi\right) A w_{N-K}, 0, \ldots, 0\right)
$$


where $\sigma_{i}$ is the $i^{\text {th }}$ diagonal entry of $\Sigma$ and $w_{i}$ is the $i^{\text {th }}$ column of $W$ (hence $W_{1}$ ). Then $X_{A}=Z V^{\top}$ solves the equation $\left(I_{N}-\Pi\right) A=X_{A}\left(I_{N}-\Pi\right)$.

$2 \Rightarrow 1$. Suppose that $\operatorname{ker}\left(I_{N}-\Pi\right)$ is not a right invariant subspace of $A$. Then there is a vector $v \in \operatorname{ker}\left(I_{N}-\Pi\right)$ such that $A v \notin \operatorname{ker}\left(I_{N}-\Pi\right)$. Invoking condition 2 we have

$$
\left(I_{N}-\Pi\right) A v=X_{A}\left(I_{N}-\Pi\right) v=0
$$

since $v \in \operatorname{ker}\left(I_{N}-\Pi\right)$. But if $A v \notin \operatorname{ker}\left(I_{N}-\Pi\right)$, then $\left(I_{N}-\Pi\right) A v \neq 0$, and we have obtained a contradiction. Thus $\operatorname{ker}\left(I_{N}-\Pi\right)$ is a right invariant subspace of $A$.

Lemma 4. Given an adjacency matrix $A$ and a permutation matrix $\Pi$ of the same dimension. The following statements are equivalent:

1) the set $\operatorname{ker}\left(I_{N}-\Pi\right)$ is a right invariant subspace of the Laplacian matrix $L=D-A$ associated to $A$;

2) there exists a solution $X_{L} \in \mathbb{R}^{N \times N}$ to the matrix equation

$$
\left(I_{N}-\Pi\right) L=X_{L}\left(I_{N}-\Pi\right) .
$$

Proof. Replace $A$ by $L$ and $X_{A}$ by $X_{L}$ in the proof of Lemma 3 .

3.4. Summary of results. For coupled systems (1), (2), combining Proposition 1, Lemma 1 and Lemma 3, we have obtained the following result.

Theorem 1. Given an adjacency matrix $A$ and a permutation matrix $\Pi$, and associate with $\Pi$ a reordering matrix $R$ such that $R^{\top} \Pi R$ is of the form (6). The following statements are equivalent:

1) the set $\operatorname{ker}\left(I_{N}-\Pi\right)$ is a right invariant subspace of $A$;

2) all blocks of the block-structured matrix (8) have constant row-sums.

3) there exists a solution $X_{A} \in \mathbb{R}^{N \times N}$ to the matrix equation

$$
\left(I_{N}-\Pi\right) A=X_{A}\left(I_{N}-\Pi\right) .
$$

If these equivalent conditions are satisfied, then $\mathcal{M}(\Pi)$ is a partial synchronization manifold for the coupled systems (1), (2).

Similarly, for coupled systems (1), (3), combining Proposition 2, Lemma 2 and Lemma 4, we have obtained the following result.

Theorem 2. Given an adjacency matrix $A$ and a permutation matrix $\Pi$, and associate with $\Pi$ a reordering matrix $R$ such that $R^{\top} \Pi R$ is of the form (6). The following statements are equivalent:

1) the set $\operatorname{ker}\left(I_{N}-\Pi\right)$ is a right invariant subspace of the Laplacian matrix $L=D-A$ associated to $A$;

2) all off-diagonal blocks of the block-structured matrix (8) have constant rowsums.

3) there exists a solution $X_{L} \in \mathbb{R}^{N \times N}$ to the matrix equation

$$
\left(I_{N}-\Pi\right) L=X_{L}\left(I_{N}-\Pi\right) .
$$

If these equivalent conditions are satisfied, then $\mathcal{M}(\Pi)$ is a partial synchronization manifold for the coupled systems (1), (3).

In both theorems, the first equivalent condition states that partially synchronized outputs generate partially synchronized inputs. Invoking this condition, one can determine permutation matrices that define a partial synchronization manifold from repeating patterns in the generalized eigenvectors of $A$ (for coupling (2)) or 
$L$ (for coupling (3)). The second equivalent condition, i.e. the row-sum condition on the blocks of the reordered adjacency matrix, has the advantage of being numerically more efficient than the first condition. In particular cases this condition can be verified even in exact arithmetic. The matrix $X_{A}$ (or $X_{L}$ ), provided in the third equivalent condition, is important for the stability of the corresponding partial synchronization manifold. In addition, if $\Pi$ and $A$ commute (or if $\Pi$ and $L$ commute), one may set $X_{A}=A$ (or $X_{L}=L$ ), cf. [32].

It is worth mentioning that the row-sum condition and its equivalent invariant subspace condition for invasive coupling (2) also appeared in a quite similar form in a paper by K. Judd [19], which was put to our attention by an anonymous reviewer. Note also that for the special case of (2) with an adjacency matrix with integer elements, one can see the row-sum condition in Proposition 2.2 of [2] as the direct analogue of the row-sum condition in Theorem 1. We remark that in the setting of [2] the row-sum condition is necessary and sufficient for the existence of the associated partial synchronization manifold, whereas in our setting the condition is only sufficient.

3.5. Example. Consider the network shown in Figure 1, which is characterized by the set of nodes

$$
\mathcal{V}=\{1,2,3,4,5,6,7,8\}
$$

edge set

$$
\begin{gathered}
\mathcal{E}=\{(1,3),(1,6),(2,7),(2,8),(3,1),(3,2),(3,4),(3,7),(4,1),(4,2),(4,3),(4,7), \\
(5,1),(5,2),(5,3),(5,4),(5,7),(6,7),(7,1),(7,2),(7,3),(7,4),(8,5)\}
\end{gathered}
$$

and adjacency matrix

$$
A=\left(\begin{array}{cccccccc}
0 & 0 & \omega_{1} & 0 & 0 & \omega_{1} & 0 & 0 \\
0 & 0 & 0 & 0 & 0 & 0 & \omega_{2} & \omega_{1} \\
\omega_{1} & \omega_{1} & 0 & \omega_{2} & 0 & 0 & \omega_{1} & 0 \\
\omega_{1} & \omega_{1} & \omega_{1} & 0 & 0 & 0 & \omega_{2} & 0 \\
\omega_{1} & \omega_{1} & \omega_{2} & \omega_{2} & 0 & 0 & \omega_{2} & 0 \\
0 & 0 & 0 & 0 & 0 & 0 & \omega_{1} & 0 \\
\omega_{1} & \omega_{1} & \omega_{1} & \omega_{2} & 0 & 0 & 0 & 0 \\
0 & 0 & 0 & 0 & \omega_{1} & 0 & 0 & 0
\end{array}\right)
$$

with positive constants $\omega_{1}$ and $\omega_{2}$. This network will be used throughout the whole text to illustrate and clarify our ideas and results.

3.5.1. Coupling (2). To simplify notation we set $\omega_{1}=1$ and $\omega_{2}=2$. (The case with general $\omega_{1}$ and $\omega_{2}$ is discussed in Sections 5 and 7.) We first try to employ the invariant subspace condition to determine a permutation matrix $\Pi$ that defined a partial synchronization manifold for the network. To that extent we determine the real Jordan canonical form of $A$ :

$$
A V=V J
$$




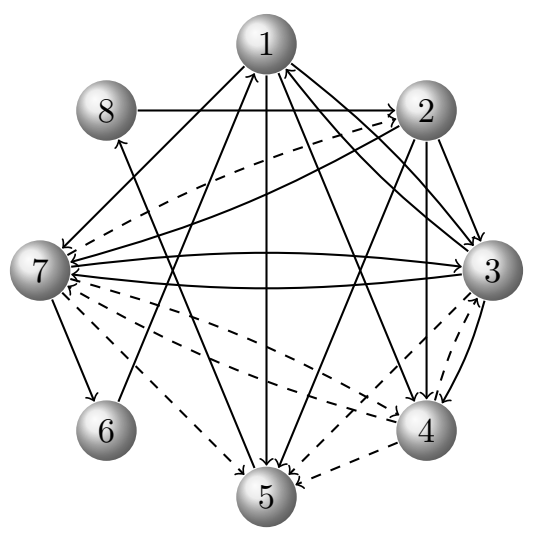

Figure 1. Example network. Solid edges have weight $\omega_{1}$, dashed edges have weight $\omega_{2}$.

with

$$
V=\left(\begin{array}{cccccccc}
0.1172 & 0.0000 & 1.8898 & -0.7071 & -1.6764 & 0.2411 & 0.1083 & 0.1216 \\
0.5274 & -0.7559 & -32.3333 & 0.7071 & 1.6764 & 0.1304 & -0.3821 & 0.2373 \\
-0.4688 & 0.1890 & 6.3825 & -0.0000 & 0.0000 & -0.1401 & 0.0511 & 0.3820 \\
0.3809 & 0.1890 & 8.0833 & -0.0000 & 0.0000 & -0.1401 & 0.0511 & 0.3820 \\
0.2344 & -0.3780 & -15.0328 & -0.0000 & 0.0000 & 0.0187 & 0.5384 & 0.6728 \\
0.2344 & -0.1890 & -8.2723 & 0.0000 & -0.7071 & 0.0529 & 0.1624 & 0.0970 \\
-0.4688 & 0.1890 & 8.0833 & -0.0000 & 0.0000 & -0.1401 & 0.0511 & 0.3820 \\
-0.1172 & 0.3780 & 15.4107 & -0.0000 & 0.7071 & 0.6173 & 0.0000 & 0.1708
\end{array}\right)
$$

and

$$
J=\left(\begin{array}{cccccccc}
-2 & & & & & & & \\
& -1 & 1 & & & & & \\
& & -1 & & & & & \\
& & & 0 & 1 & & & \\
& & & & 0 & & & \\
& & & & & 0.0303 & 0.8721 & \\
& & & & & & & 3.8721 \\
& & & & & & 0.0303 & \\
& & & & & & 3.9395
\end{array}\right)
$$

We see that the third, fourth and seventh entries of columns 2, 4, 5, 6, 7 and 8 of $V$ are identical. This suggests that the permutation matrix

$$
\Pi_{1}=\left(\begin{array}{cccccccc}
1 & 0 & 0 & 0 & 0 & 0 & 0 & 0 \\
0 & 1 & 0 & 0 & 0 & 0 & 0 & 0 \\
0 & 0 & 0 & 1 & 0 & 0 & 0 & 0 \\
0 & 0 & 0 & 0 & 0 & 0 & 1 & 0 \\
0 & 0 & 0 & 0 & 1 & 0 & 0 & 0 \\
0 & 0 & 0 & 0 & 0 & 1 & 0 & 0 \\
0 & 0 & 1 & 0 & 0 & 0 & 0 & 0 \\
0 & 0 & 0 & 0 & 0 & 0 & 0 & 1
\end{array}\right)
$$

may define a partial synchronization manifold. Indeed,

$$
\operatorname{span}\left\{V_{2}, V_{4}, V_{5}, V_{6}, V_{7}, V_{8}\right\}=\operatorname{ker}\left(I_{8}-\Pi_{1}\right),
$$


where $V_{i}$ denotes the $i^{\text {th }}$ column of $V$. Note that $\operatorname{dim} \operatorname{ker}\left(I_{8}-\Pi_{1}\right)=6$. However, as remarked before, computation of generalized eigenvectors is prone to numerical errors, hence we verify the result analytically. The vector

$$
v=\left(\begin{array}{llllllll}
v_{1} & v_{2} & v_{3} & v_{3} & v_{5} & v_{6} & v_{3} & v_{8}
\end{array}\right)^{\top} \in \operatorname{ker}\left(I_{8}-\Pi_{1}\right)
$$

and

$$
A v=\left(\begin{array}{c}
v_{3}+v_{6} \\
2 v_{3}+v_{8} \\
v_{1}+v_{2}+3 v_{3} \\
v_{1}+v_{2}+3 v_{3} \\
v_{1}+v_{2}+6 v_{3} \\
v_{3} \\
v_{1}+v_{2}+3 v_{3} \\
v_{5}
\end{array}\right) \in \operatorname{ker}\left(I_{8}-\Pi_{1}\right)
$$

for any constant $v_{1}, v_{2}, v_{3}, v_{5}, v_{6}, v_{8}$, and thus $\mathcal{M}\left(\Pi_{1}\right)$ is a partial synchronization manifold. Note that $\Pi_{1}$ partitions $\mathcal{V}$ as $\{1,2,\{3,4,7\}, 5,6,8\}$.

The same reasoning suggest that

$$
\Pi_{2}=\left(\begin{array}{cccccccc}
1 & 0 & 0 & 0 & 0 & 0 & 0 & 0 \\
0 & 1 & 0 & 0 & 0 & 0 & 0 & 0 \\
0 & 0 & 0 & 0 & 0 & 0 & 1 & 0 \\
0 & 0 & 0 & 1 & 0 & 0 & 0 & 0 \\
0 & 0 & 0 & 0 & 1 & 0 & 0 & 0 \\
0 & 0 & 0 & 0 & 0 & 1 & 0 & 0 \\
0 & 0 & 1 & 0 & 0 & 0 & 0 & 0 \\
0 & 0 & 0 & 0 & 0 & 0 & 0 & 1
\end{array}\right) \text { and } \Pi_{3}=\left(\begin{array}{cccccccc}
1 & 0 & 0 & 0 & 0 & 0 & 0 & 0 \\
0 & 1 & 0 & 0 & 0 & 0 & 0 & 0 \\
0 & 0 & 1 & 0 & 0 & 0 & 0 & 0 \\
0 & 0 & 0 & 0 & 0 & 0 & 1 & 0 \\
0 & 0 & 0 & 0 & 1 & 0 & 0 & 0 \\
0 & 0 & 0 & 0 & 0 & 1 & 0 & 0 \\
0 & 0 & 0 & 1 & 0 & 0 & 0 & 0 \\
0 & 0 & 0 & 0 & 0 & 0 & 0 & 1
\end{array}\right)
$$

may define partial synchronization manifolds. Note the third and seventh entry of column 1 of $V$ are identical, and the fourth and seventh entries of column 3 of $V$ are identical. Moreover $\operatorname{dim} \operatorname{ker}\left(I_{8}-\Pi_{2}\right)=\operatorname{dim} \operatorname{ker}\left(I_{8}-\Pi_{3}\right)=7$ and

$$
\begin{aligned}
& \operatorname{span}\left\{V_{1}, V_{2}, V_{4}, V_{5}, V_{6}, V_{7}, V_{8}\right\}=\operatorname{ker}\left(I_{8}-\Pi_{2}\right), \\
& \operatorname{span}\left\{V_{2}, V_{3}, V_{4}, V_{5}, V_{6}, V_{7}, V_{8}\right\}=\operatorname{ker}\left(I_{8}-\Pi_{3}\right) .
\end{aligned}
$$

It can easily be verified that both $\mathcal{M}\left(\Pi_{2}\right)$ and $\mathcal{M}\left(\Pi_{3}\right)$ indeed are partial synchronization manifolds, which correspond to the partitions $\{1,2,\{3,7\}, 4,5,6,8\}$ and $\{1,2,3,\{4,7\}, 5,6,8\}$ of $\mathcal{V}$, respectively.

We continue the example with verifying the row-sum condition and existence of a solution of the Sylvester equation for coupling (2) for the given adjacency matrix $A$ with $\omega_{1}=1$ and $\omega_{2}=2$ and permutation matrix $\Pi_{1}$. To test the row-sum condition on the block structured reordered adjacency matrix we define

$$
R=R_{1}=\left(\begin{array}{llllllll}
0 & 0 & 0 & 1 & 0 & 0 & 0 & 0 \\
0 & 0 & 0 & 0 & 1 & 0 & 0 & 0 \\
0 & 1 & 0 & 0 & 0 & 0 & 0 & 0 \\
1 & 0 & 0 & 0 & 0 & 0 & 0 & 0 \\
0 & 0 & 0 & 0 & 0 & 1 & 0 & 0 \\
0 & 0 & 0 & 0 & 0 & 0 & 1 & 0 \\
0 & 0 & 1 & 0 & 0 & 0 & 0 & 0 \\
0 & 0 & 0 & 0 & 0 & 0 & 0 & 1
\end{array}\right)
$$


and observe that

$$
R_{1}^{\top} \Pi_{1} R_{1}=\left(\begin{array}{ccc:c:c:c:c:c}
0 & 0 & 1 & 0 & 0 & 0 & 0 & 0 \\
1 & 0 & 0 & 0 & 0 & 0 & 0 & 0 \\
0 & 1 & 0 & 0 & 0 & 0 & 0 & 0 \\
\hdashline 0 & 0 & 0 & 1 & 0 & 0 & 0 & 0 \\
\hdashline 0 & 0 & 0 & 0 & 1 & 0 & 0 & 0 \\
\hdashline 0 & 0 & 0 & 0 & 0 & 1 & 0 & 0 \\
\hdashline 0 & 0 & 0 & 0 & 0 & 0 & 1 & 0 \\
\hdashline 0 & 0 & 0 & 0 & 0 & 0 & 0 & \frac{1}{1}
\end{array}\right) .
$$

Then we obtain

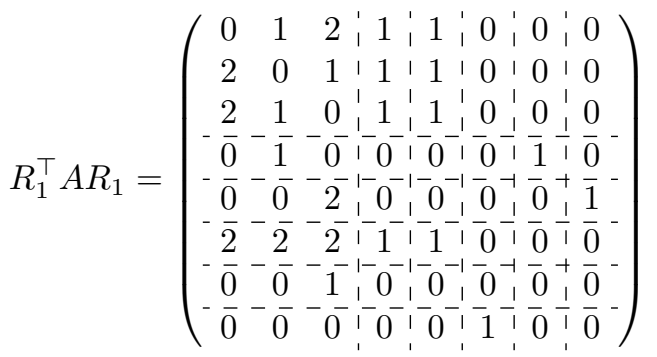

and we observe that all blocks of the reordered adjacency matrix have constant row-sums. To determine a solution $X_{A}$ to the equation

$$
\left(I_{8}-\Pi_{1}\right) A=X_{A}\left(I_{8}-\Pi_{1}\right)
$$

we employ the procedure proposed in the proof of Lemma 3 and obtain

$$
X_{A}=\left(\begin{array}{cccccccc}
0 & 0 & 0 & 0 & 0 & 0 & 0 & 0 \\
0 & 0 & 0 & 0 & 0 & 0 & 0 & 0 \\
0 & 0 & -1 & 1 & 0 & 0 & 0 & 0 \\
0 & 0 & \frac{2}{3} & -\frac{4}{3} & 0 & 0 & \frac{2}{3} & 0 \\
0 & 0 & 0 & 0 & 0 & 0 & 0 & 0 \\
0 & 0 & 0 & 0 & 0 & 0 & 0 & 0 \\
0 & 0 & \frac{1}{3} & \frac{1}{3} & 0 & 0 & -\frac{2}{3} & 0 \\
0 & 0 & 0 & 0 & 0 & 0 & 0 & 0
\end{array}\right),
$$

which indeed solves the Sylvester equation.

3.5.2. Coupling (3). Let us show that

$$
\Pi_{4}=\left(\begin{array}{llllllll}
1 & 0 & 0 & 0 & 0 & 0 & 0 & 0 \\
0 & 1 & 0 & 0 & 0 & 0 & 0 & 0 \\
0 & 0 & 0 & 1 & 0 & 0 & 0 & 0 \\
0 & 0 & 0 & 0 & 1 & 0 & 0 & 0 \\
0 & 0 & 0 & 0 & 0 & 0 & 1 & 0 \\
0 & 0 & 0 & 0 & 0 & 1 & 0 & 0 \\
0 & 0 & 1 & 0 & 0 & 0 & 0 & 0 \\
0 & 0 & 0 & 0 & 0 & 0 & 0 & 1
\end{array}\right)
$$

defines a partial synchronization manifold for the coupled systems (1), (3). The corresponding partition of $\mathcal{V}$ is $\{1,2,\{3,4,5,7\}, 6,8\}$. Again we start with the invariant subspace condition. Consider the vector

$$
v=\left(\begin{array}{llllllll}
v_{1} & v_{2} & v_{3} & v_{3} & v_{3} & v_{6} & v_{3} & v_{8}
\end{array}\right)^{\top},
$$


which, for any constant $v_{1}, v_{2}, v_{3}, v_{6}, v_{8}$, is an element of $\operatorname{ker}\left(I_{8}-\Pi_{4}\right)$. Then

$$
L v=\left(\begin{array}{cccccccc}
2 & 0 & -1 & 0 & 0 & -1 & 0 & 0 \\
0 & 3 & 0 & 0 & 0 & 0 & -2 & -1 \\
-1 & -1 & 5 & -2 & 0 & 0 & -1 & 0 \\
-1 & -1 & -1 & 5 & 0 & 0 & -2 & 0 \\
-1 & -1 & -2 & -2 & 8 & 0 & -2 & 0 \\
0 & 0 & 0 & 0 & 0 & 1 & -1 & 0 \\
-1 & -1 & -1 & -2 & 0 & 0 & 5 & 0 \\
0 & 0 & 0 & 0 & -1 & 0 & 0 & 1
\end{array}\right)\left(\begin{array}{c}
v_{1} \\
v_{2} \\
v_{3} \\
v_{3} \\
v_{3} \\
v_{6} \\
v_{3} \\
v_{8}
\end{array}\right)=\left(\begin{array}{c}
2 v_{1}-v_{3}-6 \\
3 v_{2}-2 v_{3}-v_{8} \\
-v_{1}-v_{2}+2 v_{3} \\
-v_{1}-v_{2}+2 v_{3} \\
-v_{1}-v_{2}+2 v_{3} \\
-v_{3}+v_{6} \\
-v_{1}-v_{2}+2 v_{3} \\
v_{8}-v_{3}
\end{array}\right)
$$

which clearly belongs to $\operatorname{ker}\left(I_{8}-\Pi_{4}\right)$. Thus $\mathcal{M}\left(\Pi_{4}\right)$ is a partial synchronization manifold for the coupled systems (1), (3).

For testing the row-sum condition on the reordered adjacency matrix we let

$$
R=R_{4}=\left(\begin{array}{llllllll}
0 & 0 & 0 & 0 & 1 & 0 & 0 & 0 \\
0 & 0 & 0 & 0 & 0 & 1 & 0 & 0 \\
0 & 0 & 1 & 0 & 0 & 0 & 0 & 0 \\
0 & 1 & 0 & 0 & 0 & 0 & 0 & 0 \\
1 & 0 & 0 & 0 & 0 & 0 & 0 & 0 \\
0 & 0 & 0 & 0 & 0 & 0 & 1 & 0 \\
0 & 0 & 0 & 1 & 0 & 0 & 0 & 0 \\
0 & 0 & 0 & 0 & 0 & 0 & 0 & 1
\end{array}\right)
$$

for which we obtain

$$
R_{4}^{\top} \Pi_{4} R_{4}=\left(\begin{array}{cccc:c:c:c:c}
0 & 0 & 0 & 1 & 0 & 0 & 0 & 0 \\
1 & 0 & 0 & 0 & 0 & 0 & 0 & 0 \\
0 & 1 & 0 & 0 & 0 & 0 & 0 & 0 \\
0 & 0 & 1 & 0 & 0 & 0 & 0 & 0 \\
\hdashline 0 & 0 & 0 & 1 & 0 & 0 & 0 \\
\hdashline 0 & 0 & 0 & 0 & 1 & 0 & 0 \\
\hdashline 0 & 0 & 0 & 0 & 0 & 0 & 1 & 0 \\
\hdashline 0 & 0 & 0 & 0 & 0 & 0 & 0 & 1
\end{array}\right)
$$

and

$$
R_{4}^{\top} A R_{4}=\left(\begin{array}{cccc:c:c:c:c}
0 & 2 & 2 & 2 & 1 & 1 & 0 & 0 \\
0 & 0 & 1 & 2 & 1 & 1 & 0 & 0 \\
0 & 2 & 0 & 1 & 1 & 1 & 0 & 0 \\
0 & 2 & 1 & 0 & 1 & 1 & 0 & 0 \\
\hdashline 0 & 0 & 0 & 0 & 0 & 1 & 0 \\
\hdashline 0 & 0 & 0 & 2 & 0 & 0 & 0 & 1 \\
\hdashline 0 & 0 & 0 & 1 & 0 & 0 & 0 & 0 \\
\hdashline 1 & 0 & 0 & 0 & 0 & 0 & 0 & 0
\end{array}\right)
$$

Note that all off-diagonal blocks of the reordered adjacency matrix above have indeed constant row-sums, but the block at the top-left corner has non-constant row-sums. 
Finally, using the construction explained in the proof of Lemma 3, we find that

$$
X_{L}=\left(\begin{array}{cccccccc}
0 & 0 & 0 & 0 & 0 & 0 & 0 & 0 \\
0 & 0 & 0 & 0 & 0 & 0 & 0 & 0 \\
0 & 0 & 5 & -2 & -2 & 0 & -1 & 0 \\
0 & 0 & -\frac{5}{4} & \frac{23}{4} & -\frac{9}{4} & 0 & -\frac{9}{4} & 0 \\
0 & 0 & -\frac{9}{4} & -\frac{9}{4} & \frac{23}{4} & 0 & -\frac{5}{4} & 0 \\
0 & 0 & 0 & 0 & 0 & 0 & 0 & 0 \\
0 & 0 & -\frac{3}{2} & -\frac{3}{2} & -\frac{3}{2} & 0 & \frac{9}{2} & 0 \\
0 & 0 & 0 & 0 & 0 & 0 & 0 & 0
\end{array}\right)
$$

satisfies the equation

$$
\left(I_{8}-\Pi_{4}\right) L=X_{L}\left(I_{8}-\Pi_{4}\right)
$$

\section{NECESSARY CONDITIONS FOR THE EXISTENCE OF PARTIAL SYNCHRONIZATION} MANIFOLDS

In $[39,15,20]$ necessary and sufficient conditions were presented for the existence of partial synchronization manifolds. The necessary conditions were obtained by requiring the manifold $\mathcal{M}(\Pi)$ to be forward invariant with respect to all admissible vector fields. We however consider a given node dynamics and given coupling functions (2) or (3). The sufficient conditions we presented for the existence of a partial synchronization manifold ensure that, on the manifold, systems belonging to the same cluster receive the same inputs. These conditions are not necessary as illustrated with the following example.

Example. Consider system

$$
\left\{\begin{array}{l}
\dot{x}_{i}(t)=-x_{i}(t)+\operatorname{sgn}\left(u_{i}(t)\right), \\
y_{i}(t)=x_{i}(t)
\end{array} \quad i=1, \ldots, 3,\right.
$$

where $\operatorname{sgn}(\cdot)$ is the signum function. Consider a network with adjacency matrix

$$
A=\left(\begin{array}{lll}
0 & 1 & 1 \\
2 & 0 & 0 \\
3 & 0 & 0
\end{array}\right)
$$

For

$$
\Pi=\left(\begin{array}{lll}
1 & 0 & 0 \\
0 & 0 & 1 \\
0 & 1 & 0
\end{array}\right)
$$

and corresponding partition $\mathcal{P}(\Pi)=\{\{1\},\{2,3\}\}$, subspace $\mathcal{M}(\Pi)$ is a partial synchronization manifold for both coupled system (1), (2) and (1), (3). To illustrate this, with invasive coupling (1), (2) the dynamics are described by

$$
\left\{\begin{array}{l}
\dot{x}_{1}(t)=-x_{1}(t)+\operatorname{sgn}\left(x_{2}(t-\tau)+x_{3}(t-\tau)-2 x_{1}(t)\right), \\
\dot{x}_{2}(t)=-x_{2}(t)+\operatorname{sgn}\left(2 x_{1}(t-\tau)-2 x_{2}(t)\right) \\
\dot{x}_{3}(t)=-x_{3}(t)+\operatorname{sgn}\left(3 x_{1}(t-\tau)-3 x_{3}(t)\right) .
\end{array}\right.
$$

Note that for the second and third system the value of the signum function is independent of the interaction weights. Thus we see that $\mathcal{M}(\Pi)$ is a partial synchronization manifold despite the fact that the row-sum condition on $A$ is not satisfied.

In the next two theorems we state that under mild structural conditions on the individual systems, the sufficient conditions presented in the previous section 
become necessary as well. The property of left-invertibility of system (1) plays a key role; this property is equivalent to being injective of the systems input-output map. (Note that system (9) is clearly not left-invertible.)

Theorem 3. Consider the coupled systems (1), (2) on a graph $\mathcal{G}=\mathcal{G}(\mathcal{V}, \mathcal{E}, A)$ and suppose that system (1) is left-invertible. For a given permutation matrix $\Pi$ the manifold $\mathcal{M}(\Pi)$ is a partial synchronization manifold for the coupled systems (1), (2) if and only if the following equivalent conditions hold true:

1) the set $\operatorname{ker}\left(I_{N}-\Pi\right)$ is a right invariant subspace of $A$;

2) all blocks of the block-structured matrix (8), where reordering matrix $R$ such that $R^{\top} \Pi R$ is of the form (6), have constant row-sums;

3) there exists a solution $X_{A} \in \mathbb{R}^{N \times N}$ to the matrix equation

$$
\left(I_{N}-\Pi\right) A=X_{A}\left(I_{N}-\Pi\right)
$$

Theorem 4. Consider the coupled systems (1), (3) on a graph $\mathcal{G}=\mathcal{G}(\mathcal{V}, \mathcal{E}, A)$ and suppose that system (1) is left-invertible. For a given permutation matrix $\Pi$ the manifold $\mathcal{M}(\Pi)$ is a partial synchronization manifold for the coupled systems (1), (3) if and only if the following equivalent conditions hold true:

1) the set $\operatorname{ker}\left(I_{N}-\Pi\right)$ is a right invariant subspace of the Laplacian matrix $L=D-A$ associated to $A$;

2) all off-diagonal blocks of the block-structured matrix (8), where reordering matrix $R$ such that $R^{\top} \Pi R$ is of the form (6), have constant row-sums;

3) there exists a solution $X_{L} \in \mathbb{R}^{N \times N}$ to the matrix equation

$$
\left(I_{N}-\Pi\right) L=X_{L}\left(I_{N}-\Pi\right) .
$$

Proof. Sufficiency follows from Theorems 1 and 2. The proof of necessity in both theorems is by contradiction. Assume that the row-sum condition is not satisfied. Then we can always choose initial data for the coupled systems, lying on $\mathcal{M}(\Pi)$ and such at least two systems within the same cluster receive a different input at the starting time. By the left-invertibility different inputs imply different outputs, hence that solution cannot stay on $\mathcal{M}(\Pi)$.

In light of the discussion above, one can always construct vector fields $f$ and $h$ such that system (1) is left-invertible. Thus, the conditions presented in Theorems 1 and 2 would be necessary and sufficient if one adopts the definition of a partial synchronization as used in $[39,15,20]$.

Necessary and sufficient conditions for certain classes of systems (1) to be leftinvertible are found in $[18,25]$. Because we wish not to introduce additional notation, we discuss conditions left-invertibility only for a special case of systems (1). Consider (1) with

$$
f\left(x_{i}(t), u_{i}(t)\right)=\tilde{f}\left(x_{i}(t)\right)+B u_{i}(t)
$$

and

$$
h\left(x_{i}(t)\right)=C x_{i}(t)
$$

where $\tilde{f}: \mathbb{R}^{n} \rightarrow \mathbb{R}^{n}$ is smooth and matrices $B \in \mathbb{R}^{n \times m}, C \in \mathbb{R}^{m \times n}$. For this class of systems an easily verifiable condition for left-invertibility is that

$$
\operatorname{rank}(C B)=m
$$


cf. [17] pp. 342. Indeed, one can show that if the matrix $C B$ has full rank, then there exists a well-defined change of coordinates (cf. $[8,31]$ )

$$
x_{i}(t) \mapsto\left(z_{i}(t), y_{i}(t)\right),
$$

with $z_{i}(t) \in \mathbb{R}^{n-m}$ and $y_{i}(t) \in \mathbb{R}^{m}$, such that the systems dynamics in these new coordinates read

$$
\left\{\begin{array}{l}
\dot{z}_{i}(t)=q\left(z_{i}(t), y_{i}(t)\right) \\
\dot{y}_{i}(t)=a\left(z_{i}(t), y_{i}(t)\right)+C B u_{i}(t)
\end{array}\right.
$$

where $q: \mathbb{R}^{n-m} \times \mathbb{R}^{m} \rightarrow \mathbb{R}^{n-m}$ and $a: \mathbb{R}^{n-m} \times \mathbb{R}^{m} \rightarrow \mathbb{R}^{m}$ are smooth functions; Since $C B$ has full rank it is clear that for systems (10) the outputs can only remain identical if their inputs are the same.

\section{Decomposition of GRAPhS}

In practical applications it is no exception that there are physically distinct communication channels, which each have their specific properties such as a characteristic time-delay and/or characteristic interaction weights. In this section we discuss the latter case; the multiple time-delay case is considered in Section 6.

Suppose that a given network $\mathcal{G}$ with $r$ characteristic scalar interaction weights $\omega_{\ell}, \ell=1, \ldots, r$, decomposes as

$$
\mathcal{G}(\mathcal{V}, \mathcal{E}, A)=\mathcal{G}_{1}\left(\mathcal{V}, \mathcal{E}_{1}, \omega_{1} A_{1}\right) \oplus \cdots \oplus \mathcal{G}_{r}\left(\mathcal{V}, \mathcal{E}_{r}, \omega_{r} A_{r}\right)
$$

where $A_{\ell}$ are adjacency matrices with non-negative integer coefficients and $\mathcal{E}_{\ell}$ is the set of edges corresponding to $A_{\ell}$ (that is, $(i, j) \in \mathcal{E}_{\ell}$ if and only if the $i j^{\text {th }}$ entry of $\left.A_{\ell}>0\right)$. Here $\oplus$ denotes a sum on graphs with the same set of nodes $\mathcal{V}$, which joins the sets of edges and sums the adjacency matrices. Thus for two graphs $\mathcal{G}_{i}\left(\mathcal{V}, \mathcal{E}_{i}, A_{i}\right)$ and $\mathcal{G}_{j}\left(\mathcal{V}, \mathcal{E}_{j}, A_{j}\right)$ their graph sum $\mathcal{G}_{i}\left(\mathcal{V}, \mathcal{E}_{i}, A_{i}\right) \oplus \mathcal{G}_{j}\left(\mathcal{V}, \mathcal{E}_{j}, A_{j}\right)$ is the graph with set of nodes $\mathcal{V}$, set of edges $\mathcal{E}_{1} \cup \mathcal{E}_{2}$ and adjacency matrix $A_{1}+A_{2}$. Hence

$$
\mathcal{G}_{1}\left(\mathcal{V}, \mathcal{E}_{1}, \omega_{1} A_{1}\right) \oplus \cdots \oplus \mathcal{G}_{r}\left(\mathcal{V}, \mathcal{E}_{r}, \omega_{r} A_{r}\right)=\mathcal{G}\left(\mathcal{V}, \cup_{\ell=1}^{r} \mathcal{E}_{\ell}, \sum_{\ell=1}^{r} \omega_{\ell} A_{\ell}\right)
$$

such that

$$
A=\omega_{1} A_{1}+\omega_{2} A_{2}+\ldots+\omega_{r} A_{r}
$$

and

$$
\mathcal{E}=\cup_{\ell=1}^{r} \mathcal{E}_{\ell} .
$$

We assume that none of the subgraphs $\mathcal{G}_{\ell}$ be an empty graph (i.e. an edgeless graph). However, we remark that

- any subgraph $\mathcal{G}_{\ell}\left(\mathcal{V}, \mathcal{E}_{\ell}, \omega_{\ell} A_{\ell}\right)$ may not be strongly connected;

- any two subgraphs $\mathcal{G}_{i}$ and $\mathcal{G}_{j}$ may have common edges.

In what follows we call the numbers $\omega_{\ell}, \ell=1, \ldots, r$ the basis weights.

Consider any adjacency matrix $A_{\ell}, \ell=1, \ldots, r$, and let $\Pi$ be a permutation matrix of appropriate dimension. Associate with that $\Pi$ a reordering matrix $R$ such that $R^{\top} \Pi R$ is of the form (6). Then Theorem 1 implies that the following statements are equivalent:

1) $\operatorname{ker}\left(I_{N}-\Pi\right)$ is a right invariant subspace of matrix $A_{\ell}$; 
2) all blocks of the block-structured matrix

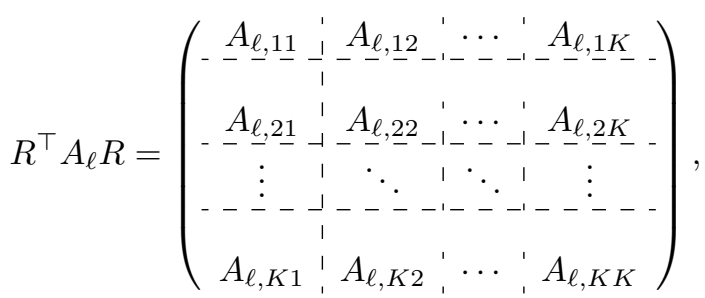

with $A_{\ell, i j} \in \mathbb{R}^{k_{i} \times k_{j}}$ have constant row-sums;

3) there exist a matrix $X_{A_{\ell}}$ such that

$$
(I-\Pi) A_{\ell}=X_{A_{\ell}}(I-\Pi) .
$$

It readily follows that if the permutation matrix $\Pi$ is such that the conditions above hold true for each matrix $A_{\ell}, \ell=1, \ldots, r$, then $\mathcal{M}(\Pi)$ is a partial synchronization manifold for the coupled systems (1), (2).

Likewise, Theorem 2 implies that the following statements are equivalent:

1) $\operatorname{ker}\left(I_{N}-\Pi\right)$ is a right invariant subspace of the Laplacian matrix $L_{\ell}=$ $D_{\ell}-A_{\ell}$ associated to $A_{\ell}$, where

$$
D_{\ell}=\left(\begin{array}{lll}
\sum_{j=2}^{N} a_{\ell, 1 j} & & \\
& \ddots & \\
& & \sum_{j=1}^{N-1} a_{\ell, N j}
\end{array}\right) ;
$$

2) all off-diagonal blocks of the block-structured matrix (13) have constant row-sums;

3) there exist a matrix $X_{L_{\ell}}$ such that

$$
(I-\Pi) L_{\ell}=X_{L_{\ell}}(I-\Pi) .
$$

It follows, again, that if the permutation matrix $\Pi$ is such that the conditions above hold true for each matrix $A_{\ell}, \ell=1, \ldots, r$, then $\mathcal{M}(\Pi)$ is a partial synchronization manifold for the coupled systems (1), (3).

In other words, if a permutation matrix $\Pi$ defines a partial synchronization manifold for each subgraph $\mathcal{G}_{\ell}\left(\mathcal{V}, \mathcal{E}_{\ell}, \omega_{\ell} A_{\ell}\right), \ell=1, \ldots, r$, then that $\Pi$ defines a partial synchronization manifold for the whole network $\mathcal{G}(\mathcal{V}, \mathcal{E}, A)$. However, as we show in the example at the end of this section, it is possible to construct

$$
\mathcal{G}(\mathcal{V}, \mathcal{E}, A)=\mathcal{G}_{1}\left(\mathcal{V}, \mathcal{E}_{1}, \omega_{1} A_{1}\right) \oplus \cdots \oplus \mathcal{G}_{r}\left(\mathcal{V}, \mathcal{E}_{r}, \omega_{r} A_{r}\right)
$$

for which there is a permutation matrix $\Pi$ such that $\mathcal{M}(\Pi)$

- is a partial synchronization manifold for $\mathcal{G}$;

- is not a partial synchronization manifold for each $\operatorname{subgraph} \mathcal{G}_{\ell}\left(\mathcal{V}, \mathcal{E}_{\ell}, \omega_{\ell} A_{\ell}\right)$, $\ell=1, \ldots, r$.

A condition for which the partial synchronization manifolds for $\mathcal{G}(\mathcal{V}, \mathcal{E}, A)$ are equal to the common partial synchronization manifolds for all subgraphs $\mathcal{G}_{\ell}\left(\mathcal{V}, \mathcal{E}_{\ell}, \omega_{\ell} A_{\ell}\right)$, $\ell=1, \ldots, r$, is provided below.

Lemma 5. Suppose that in decomposition (12) the numbers $\omega_{\ell}$ are rationally independent, i.e., for integer numbers $q_{1}, \ldots, q_{r}$ the implication

$$
\sum_{\ell=1}^{r} \omega_{\ell} q_{\ell}=0 \Rightarrow q_{\ell}=0 \quad \forall \ell=1, \ldots, r
$$


holds. Suppose there is a permutation matrix $\Pi$ of appropriate dimension and associate with this matrix $\Pi$ a reordering matrix $R$ such that $R^{\top} \Pi R$ is of the form (6). Then the $i j^{\text {th }}$ block of matrix (8) has constant row-sums if and only if for each $\ell=1, \ldots, r$ the $i j^{\text {th }}$ block of matrix (13) has constant row-sums.

Proof. We assume without loss of generality that we have $R^{\top} \Pi R$ of the form (6) such that $R=I_{N}$. It is clear that all blocks $A_{1, i j}, \ldots, A_{r, i j}$ having constant rowsums implies that $A_{i j}$ has constant row-sums. To prove the opposite direction, consider any block $A_{i j}$ of matrix $A$. In case all row-sums of that block are zero we should have all row-sums of the blocks $A_{1, i j}, \ldots, A_{r, i j}$ all being zero. Consider the case that (some of) the row-sums of that block $A_{i j}$ are non-zero. Our claim is that each such block $A_{i j}$ can be written as

$$
A_{i j}=\omega_{1} A_{1, i j}+\ldots+\omega_{r} A_{r, i j}
$$

where each block $A_{\ell, i j}$ of $A_{\ell}$ has constant row sums. Let $\rho_{\ell}$ be a vector that contains the row-sums of $A_{\ell, i j}$, i.e. $\rho_{\ell}=A_{\ell, i j} \mathbf{1}$. Suppose that the row-sums of at least one block $A_{\ell, i j}$ are not constant. The assumption that $A_{i j}$ has constant row-sums implies that

$$
\omega_{1} \rho_{1, i}+\omega_{2} \rho_{2, i}+\ldots+\omega_{r} \rho_{r, i}=\omega_{1} \rho_{1, j}+\omega_{2} \rho_{2, j}+\ldots+\omega_{r} \rho_{r, j}
$$

for every $i$ and $j$, where $\rho_{\ell, i}$ is the $i^{\text {th }}$ entry of the vector $\rho_{\ell}$. Rewrite the latter equation as

$$
\omega_{1}\left(\rho_{1, i}-\rho_{1, j}\right)+\omega_{2}\left(\rho_{2, i}-\rho_{2, j}\right)+\ldots+\omega_{r}\left(\rho_{r, i}-\rho_{r, j}\right)=0 .
$$

Because each $\rho_{\ell, i}-\rho_{\ell, j}$ is an integer by construction, the last equation can only hold true if some of the numbers $\omega_{\ell}$ are rationally dependent. Thus we have obtained a contradiction, which proves that the row-sums of all block $A_{\ell, i j}$ are constant.

Theorem 5. Consider a graph $\mathcal{G}$ that decomposes as (11) and suppose that the numbers $\omega_{1}, \ldots, \omega_{r}$ are rationally independent. Given a permutation matrix $\Pi$ of appropriate dimension and associate with that $\Pi$ a reordering matrix $R$ such that $R^{\top} \Pi R$ is of the form (6). Then the following statements are equivalent to 1), 2) and 3) of Theorem 1:

$\left.1^{\prime}\right)$ for each $\ell=1, \ldots, r$, $\operatorname{ker}\left(I_{N}-\Pi\right)$ is a right invariant subspace of matrix $A_{\ell}$

$\left.2^{\prime}\right)$ for each $\ell=1, \ldots, r$, all blocks of the block-structured matrix (13) have constant row-sums;

$\left.3^{\prime}\right) X_{A}=\omega_{1} X_{A_{1}}+\ldots+\omega_{r} X_{A_{r}}$ where, for each $\ell=1, \ldots, r, X_{A_{\ell}}$ solves

$$
(I-\Pi) A_{\ell}=X_{A_{\ell}}(I-\Pi) \text {. }
$$

If the equivalent conditions above hold true, then the conclusion of Theorem 1 applies. If, in addition, the systems (1) are left-invertible, then the conclusion of Theorem 3 holds true.

Theorem 6. Consider a graph $\mathcal{G}$ that decomposes as (11) and suppose that the numbers $\omega_{1}, \ldots, \omega_{r}$ are rationally independent. Given a permutation matrix $\Pi$ of appropriate dimension and associate with that $\Pi$ a reordering matrix $R$ such that $R^{\top} \Pi R$ is of the form (6). Then the following statements are equivalent to 1), 2) and 3) of Theorem 2:

$\left.1^{\prime}\right)$ for each $\ell=1, \ldots, r, \operatorname{ker}\left(I_{N}-\Pi\right)$ is a right invariant subspace of the Laplacian matrix $L_{\ell}$ associated to $A_{\ell}$; 
$\left.2^{\prime}\right)$ for each $\ell=1, \ldots, r$, all off-diagonal blocks of the block-structured matrix (13) have constant row-sums;

$\left.3^{\prime}\right) X_{L}=\omega_{1} X_{L_{1}}+\ldots+\omega_{r} X_{L_{r}}$ where, for each $\ell=1, \ldots, r, X_{L_{\ell}}$ solves

$$
(I-\Pi) L_{\ell}=X_{L_{\ell}}(I-\Pi) \text {. }
$$

If the equivalent conditions above hold true, then the conclusion of Theorem 2 applies. If, in addition, the systems (1) are left-invertible, then the conclusion of Theorem 4 holds true.

Remark 2. If the weights $\left(\omega_{1}, \ldots, \omega_{r}\right)$ are rationally dependent, there always exists an integer $p<r$ and a matrix $\Gamma \in \mathbb{Z}^{r \times p}$ of full column rank such that

$$
\left[\begin{array}{c}
\omega_{1} \\
\vdots \\
\omega_{r}
\end{array}\right]=\Gamma\left[\begin{array}{c}
s_{1} \\
\vdots \\
s_{p}
\end{array}\right],
$$

with the numbers $s_{1}, \ldots, s_{p}$ rationally independent, see [23, Appendix A]. Thus, rationally dependent numbers depend on a smaller number of rationally independent numbers. As a consequence there exist matrices $A_{1}^{*}, \ldots, A_{p}^{*}$ with integer elements such that

$$
A=\sum_{\ell=1}^{r} A_{\ell} \omega_{\ell}=\sum_{\ell=1}^{p} A_{\ell}^{*} s_{\ell} .
$$

5.1. Balanced coloring of graphs. For networks of systems with delay-free coupling a nice graphical procedure is developed for testing the existence of partial synchronization manifolds. More precisely, the existence of a partial synchronization manifold is implied by the existence of balanced equivalence relations on the nodes of the network, which is equivalent to the existence of a so-called balanced coloring of the nodes of the network [39, 15, 20].

In what follows we adapt the definition of a balanced coloring to the problem under consideration (delayed interconnections, diffusive coupling, weighted adjacency matrix) and we show that the conditions for a partial synchronization manifold derived in the previous section are equivalent to the existence of a balanced coloring, under the assumption that $\mathcal{G}$ decomposes as in (11)-(12) with the numbers $\omega_{\ell}$, $\ell=1, \ldots, r$, being all rationally independent.

More precisely, we associate with $\mathcal{G}$ and the decomposition (12) the multi-graph ${ }^{1}$

$$
\overline{\mathcal{G}}=(\mathcal{V}, \overline{\mathcal{E}})
$$

where $\overline{\mathcal{E}}=\sqcup_{\ell=1}^{r} \overline{\mathcal{E}}_{\ell}$, with $\sqcup$ denoting the disjoint union of the multi-sets ${ }^{2} \overline{\mathcal{E}}_{\ell}, \ell \in$ $\{1, \ldots, r\}$ whose number, heads and tails are defined by the nonzero entries of $A_{\ell}$. More precisely, if the $i j^{\text {th }}$ entry of $A_{\ell}$ is $p$, which is an integer by definition, then the multi-set $\overline{\mathcal{E}}_{\ell}$ contains $p$ edges $(i, j)$. We shall refer to edges in a multi-set $\overline{\mathcal{E}}_{\ell}$ as edges of type $\ell$. The reason for taking the disjoint union (rather than the union) for construction of $\overline{\mathcal{E}}$ is that we shall need to retrieve from $\overline{\mathcal{E}}$ not only the existence of edges but also their type.

Definition 2 (Balanced $K$-coloring). Consider a graph $\mathcal{G}=(\mathcal{V}, \mathcal{E}, A)$ with adjacency matrix $A$ that decomposes as in (12). Let $\overline{\mathcal{G}}$ be the multi-graph (14) associated

\footnotetext{
${ }^{1} \mathrm{~A}$ multi-graph is a graph in which pairs of nodes may be joined by multiple edges.

${ }^{2} \mathrm{~A}$ multi-set is a set in which elements are allowed to occur more than once.
} 
to $\mathcal{G}$ and (12). A coloring of the nodes $\mathcal{V}$ with $K$ distinct colors $c_{1}, \ldots, c_{K}$ is a balanced $K$-coloring of $\overline{\mathcal{G}}$ if and only if for each $i=1,2 \ldots, K$, for each $j=1,2, \ldots, K$, and for each $\ell=1, \ldots, r$ every $c_{i}$-colored node receives the same number of edges of type $\ell$ from $c_{j}$-colored nodes.

Remark 3. In the above definition we make a distinction between edges of different type, i.e., in the description of the network we assume non-trivial equivalence relations on the edges. The setting of [15] is more general in the sense that one can start from any equivalence relation on the nodes as well. This is not necessary in our setting, as we assume that all systems associated to the nodes to be identical.

Corollary 1. Consider a graph $\mathcal{G}=(\mathcal{V}, \mathcal{E}, A)$ that decomposes as in (11) with all matrices $A_{\ell}$ having non-negative integer entries and the numbers $\omega_{\ell}, \ell=1, \ldots, r$, being all rationally independent. Let $\overline{\mathcal{G}}$ be the multi-graph (14) associated to $\mathcal{G}$ and (12). Then conditions 1') - 3') of Theorem 5 (and conditions 1) - 3) of Theorem 1)) hold if and only if the coloring of the nodes, according to the partition induced by $\Pi$, is a balanced $K$-coloring of $\overline{\mathcal{G}}$.

Proof. Take any $\ell \in\{1, \ldots, r\}$. We shall prove that the following statements are equivalent:

a. the coloring of $\overline{\mathcal{G}}_{\ell}$, induced by $\Pi$, is a balanced $K$-coloring;

b. every block of (13), where $R$ is associated with $\Pi$, has constant row-sums.

(b. $\Rightarrow$ a.) Without loss of generality we assume that the systems have been relabeled such that $\Pi$ has the form (6) and the matrix $A_{\ell}$ has the form (13) (i.e. $\left.R=I_{N}\right)$. Assign the nodes $1, \ldots, k_{1}$ color $c_{1}$, nodes $k_{1}+1, \ldots, k_{1}+k_{2}$ color $c_{2}$, and so on. The assumption of constant row-sums of each block $A_{\ell, i j}$ implies that every node of color $c_{i}$ receives the same number of edges from $c_{j}$-colored nodes. (Recall that the entries of $A_{\ell}$ count the number of edges between the corresponding nodes.) Thus the coloring of the nodes is a balanced $K$-coloring of $\overline{\mathcal{G}}_{\ell}$.

(a. $\Rightarrow$ b.) Without loss of generality we assume that the nodes of $\overline{\mathcal{G}}_{\ell}$ have been relabeled such that nodes $1, \ldots, k_{1}$ have color $c_{1}$, nodes $k_{1}+1, \ldots, k_{1}+k_{2}$ have color $c_{2}$, and so on. Note that the matrix $A_{\ell}$ associated to this relabeled multi-graph $\overline{\mathcal{G}}_{\ell}$ has the block structure (13). Suppose a. holds true but there is a block $A_{\ell, i j}$ with non-constant row-sums. Then there are two $c_{i}$-colored nodes that receive a different number of edges from $c_{j}$-colored nodes. But then the above coloring of $\overline{\mathcal{G}}_{\ell}$ is not balanced $K$. Hence, by contradiction we conclude that the row-sums of each block $A_{\ell, i j}$ need to be constant.

Remark 4. Theorem 5 and Corollary 1 state, in essence, that the problem of finding the partial synchronization manifolds of a network with graph $\overline{\mathcal{G}}$ (and the original network (1)-(2) if the basis weights are rationally independent) is equivalent to finding the common partial synchronization manifolds of networks with the same nodes and graphs $\mathcal{G}_{\ell}, \ell=1, \ldots, r$. In [1] a similar result was obtained.

The condition of existence of a balanced $K$-coloring is too restrictive for the existence of a partial synchronization manifold $\mathcal{M}(\Pi)$ in networks of coupled systems (1), (3). Because coupling (3) is non-invasive, $\mathcal{M}(\Pi)$ can be a partial synchronization manifold even if the number of edges (of any type) between nodes within the same cluster is not constant, since the corresponding coupling terms in (3) vanish. This property motives us to introduce the notion of relaxed balanced $K$-coloring. 
Definition 3 (Relaxed balanced $K$-coloring). Consider a graph $\mathcal{G}=(\mathcal{V}, \mathcal{E}, A)$ with adjacency matrix $A$ that decomposes as in (12). Let $\overline{\mathcal{G}}$ be the multi-graph (14) associated to $\mathcal{G}$ and (12).

A coloring of the nodes $\mathcal{V}$ with $K$ distinct colors $c_{1}, \ldots, c_{K}$ is a relaxed balanced $K$-coloring of $\overline{\mathcal{G}}$ if and only if for each $i=1,2 \ldots, K$, for each $j=1,2, \ldots, K$ with $j \neq i$, and for each $\ell=1, \ldots, r$, every $c_{i}$-colored node receives the same number of edges of type $\ell$ from $c_{j}$-colored nodes.

Notice that the difference with the definition of a balanced coupling lies in the condition $i \neq j$.

We remark that, by definition, every balanced $K$-coloring is a relaxed balanced $K$-coloring. In addition we remark that the relaxed balanced 1-coloring, i.e. the coloring of all nodes with the same color, always exists; We refer to the relaxed balanced 1-coloring as the trivial relaxed balanced coloring.

The next result is the counterpart of Corollary 1.

Corollary 2. Consider a graph $\mathcal{G}=(\mathcal{V}, \mathcal{E}, A)$ that decomposes as in (11) with all matrices $A_{\ell}$ having non-negative integer entries and the numbers $\omega_{\ell}, \ell=1, \ldots, r$, being all rationally independent. Let $\overline{\mathcal{G}}$ be the multi-graph (14) associated to $\mathcal{G}$ and (12). Then conditions 1') - 3') of Theorem 6 (and conditions 1)-3) of Theorem 2) hold if and only if the coloring of the nodes, according to the partition induced by $\Pi$, is a relaxed balanced $K$-coloring of $\overline{\mathcal{G}}$.

Proof. The proof is analogous to the proof of Corollary 1 with the exception that for a relaxed balanced $K$-coloring the number of edges that nodes of color $c_{i}$ receive from nodes of the same color does not need to be constant (by definition), or, equivalently, the diagonal blocks of (13) do not need to have constant row-sums.

5.2. Example. We consider again the network presented in Section 3.5. This network decomposes as

$$
\mathcal{G}(\mathcal{V}, \mathcal{E}, A)=\mathcal{G}_{1}\left(\mathcal{V}, \mathcal{E}_{1}, \omega_{1} A_{1}\right) \oplus \mathcal{G}_{2}\left(\mathcal{V}, \mathcal{E}_{2}, \omega_{2} A_{2}\right)
$$

where

$$
\begin{aligned}
& \mathcal{E}_{1}=\{(1,3),(1,6),(2,8),(3,1),(3,2),(3,7),(4,1),(4,2),(4,3),(4,7),(5,1),(5,2),(6,7),(7,1),(7,2),(7,3),(8,5)\}, \\
& \mathcal{E}_{2}=\{(2,7),(3,4),(5,3),(5,4),(5,7),(7,4)\},
\end{aligned}
$$

$$
A_{1}=\left(\begin{array}{cccccccc}
0 & 0 & 1 & 0 & 0 & 1 & 0 & 0 \\
0 & 0 & 0 & 0 & 0 & 0 & 0 & 1 \\
1 & 1 & 0 & 0 & 0 & 0 & 1 & 0 \\
1 & 1 & 1 & 0 & 0 & 0 & 0 & 0 \\
1 & 1 & 0 & 0 & 0 & 0 & 0 & 0 \\
0 & 0 & 0 & 0 & 0 & 0 & 1 & 0 \\
1 & 1 & 1 & 0 & 0 & 0 & 0 & 0 \\
0 & 0 & 0 & 0 & 1 & 0 & 0 & 0
\end{array}\right) \text { and } A_{2}=\left(\begin{array}{cccccccc}
0 & 0 & 0 & 0 & 0 & 0 & 0 & 0 \\
0 & 0 & 0 & 0 & 0 & 0 & 1 & 0 \\
0 & 0 & 0 & 1 & 0 & 0 & 0 & 0 \\
0 & 0 & 0 & 0 & 0 & 0 & 1 & 0 \\
0 & 0 & 1 & 1 & 0 & 0 & 1 & 0 \\
0 & 0 & 0 & 0 & 0 & 0 & 0 & 0 \\
0 & 0 & 0 & 1 & 0 & 0 & 0 & 0 \\
0 & 0 & 0 & 0 & 0 & 0 & 0 & 0
\end{array}\right)
$$


Let us assume for the moment that $\omega_{1}$ and $\omega_{2}$ are rationally independent and consider the permutation matrix

$$
\Pi_{1}=\left(\begin{array}{cccccccc}
1 & 0 & 0 & 0 & 0 & 0 & 0 & 0 \\
0 & 1 & 0 & 0 & 0 & 0 & 0 & 0 \\
0 & 0 & 0 & 1 & 0 & 0 & 0 & 0 \\
0 & 0 & 0 & 0 & 0 & 0 & 1 & 0 \\
0 & 0 & 0 & 0 & 1 & 0 & 0 & 0 \\
0 & 0 & 0 & 0 & 0 & 1 & 0 & 0 \\
0 & 0 & 1 & 0 & 0 & 0 & 0 & 0 \\
0 & 0 & 0 & 0 & 0 & 0 & 0 & 1
\end{array}\right)
$$

which was introduced in Section 3.5. We will show that this matrix $\Pi_{1}$ defines a partial synchronization manifold for the coupled systems (1), (2) (and, of course, also for coupled systems (1), (3)).

First we verify the invariant subspace condition. Let

$$
v=\left(\begin{array}{llllllll}
v_{1} & v_{2} & v_{3} & v_{3} & v_{5} & v_{6} & v_{3} & v_{8}
\end{array}\right)
$$

with any real constants $v_{1}, v_{2}, v_{3}, v_{5}, v_{6}, v_{8}$ such that $v \in \operatorname{ker}\left(I_{8}-\Pi_{i}\right)$. Then

$$
A_{1} v=\left(\begin{array}{c}
v_{3}+v_{6} \\
v_{8} \\
v_{1}+v_{2}+v_{3} \\
v_{1}+v_{2}+v_{3} \\
v_{1}+v_{2} \\
v_{3} \\
v_{1}+v_{2}+v_{3} \\
v_{5}
\end{array}\right) \in \operatorname{ker}\left(I_{8}-\Pi_{1}\right), \quad A_{2} v=\left(\begin{array}{c}
0 \\
v_{8} \\
v_{3} \\
v_{3} \\
3 v_{3} \\
0 \\
v_{3} \\
0
\end{array}\right) \in \operatorname{ker}\left(I_{8}-\Pi_{1}\right)
$$

hence $\operatorname{ker}\left(I_{8}-\Pi_{1}\right)$ is a right invariant subspace of $\omega_{1} A_{1}+\omega_{2} A_{2}$. Thus $\mathcal{M}\left(\Pi_{1}\right)$ is a partial synchronization manifold for the network of coupled systems.

Next we consider the matrix $R_{1}$ given in Section 3.5 for which

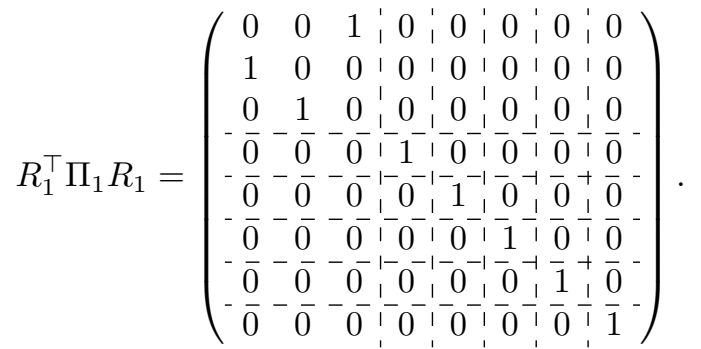

Then we obtain

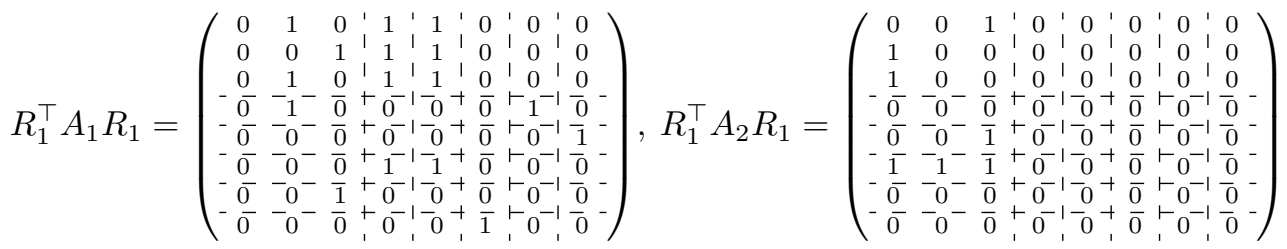

and it follows that all blocks of

$$
R_{1}^{\top} A R_{1}=R_{1}^{\top}\left(\omega_{1} A_{1}+\omega_{2} A_{2}\right) R_{1}
$$


have constant row-sums. Moreover, matrices

$$
X_{A_{1}}=\left(\begin{array}{cccccccc}
0 & 0 & 0 & 0 & 0 & 0 & 0 & 0 \\
0 & 0 & 0 & 0 & 0 & 0 & 0 & 0 \\
0 & 0 & 0 & 0 & 0 & 0 & 0 & 0 \\
0 & 0 & -\frac{1}{3} & -\frac{1}{3} & 0 & 0 & \frac{2}{3} & 0 \\
0 & 0 & 0 & 0 & 0 & 0 & 0 & 0 \\
0 & 0 & 0 & 0 & 0 & 0 & 0 & 0 \\
0 & 0 & \frac{1}{3} & \frac{1}{3} & 0 & 0 & -\frac{2}{3} & 0 \\
0 & 0 & 0 & 0 & 0 & 0 & 0 & 0
\end{array}\right), X_{A_{2}}=\left(\begin{array}{cccccccc}
0 & 0 & 0 & 0 & 0 & 0 & 0 & 0 \\
0 & 0 & 0 & 0 & 0 & 0 & 0 & 0 \\
0 & 0 & 0 & 0 & 0 & 0 & 0 & 0 \\
0 & 0 & -\frac{1}{3} & \frac{2}{3} & 0 & 0 & -\frac{1}{3} & 0 \\
0 & 0 & \frac{1}{3} & -\frac{2}{3} & 0 & 0 & \frac{1}{3} & 0 \\
0 & 0 & 0 & 0 & 0 & 0 & 0 & 0 \\
0 & 0 & 0 & 0 & 0 & 0 & 0 & 0 \\
0 & 0 & 0 & 0 & 0 & 0 & 0 & 0
\end{array}\right)
$$

solve the equations

$$
\begin{aligned}
& \left(I_{8}-\Pi_{1}\right) A_{1}=X_{A_{1}}\left(I_{8}-\Pi_{1}\right), \\
& \left(I_{8}-\Pi_{1}\right) A_{2}=X_{A_{2}}\left(I_{8}-\Pi_{1}\right),
\end{aligned}
$$

and it is straightforward to verify that

$$
\left(I_{8}-\Pi_{1}\right) A=\left(I_{8}-\Pi_{1}\right)\left(\omega_{1} A_{1}+\omega_{2} A_{2}\right)=\left(\omega_{1} X_{A_{1}}+\omega_{2} X_{A_{2}}\right)\left(I_{8}-\Pi_{1}\right)=X_{A}\left(I_{8}-\Pi_{1}\right) .
$$

For finding additional partial synchronization manifolds for the coupled systems (1), (2) or (1), (3) we have identified the (relaxed) balanced $K$-colorings of the graphs $\overline{\mathcal{G}}$ associated with $\mathcal{G}$ and with the decomposition

$$
A=\omega_{1} A_{1}+\omega_{2} A_{2}
$$

Figure 2 shows all balanced $K$-colorings and relaxed balanced $K$-colorings for the example network with $\omega_{1}$ and $\omega_{2}$ being rationally independent. In this figure the solid edges are the edges related to $A_{1}$ and the dashed edges are the edges related to $A_{2}$. To make it easier to verify if the coloring is a (relaxed) balanced $K$-coloring we have assigned the edges the color of the nodes at their tail.

The colorings shown in Figure 2 correspond to the following partitions of $\mathcal{V}$ :
(a) $\mathcal{P}=\{\{1\},\{2\},\{3,4,7\},\{5\},\{6\},\{8\}\}$
(b) $\mathcal{P}=\{\{1\},\{2\},\{3,7\},\{4\},\{5\},\{6\},\{8\}\}$
(c) $\mathcal{P}=\{\{1\},\{2\},\{3\},\{4,7\},\{5\},\{6\},\{8\}\}$
(d) $\mathcal{P}=\{\{1\},\{2\},\{3,5,7\},\{4\},\{6,8\}\}$
(e) $\mathcal{P}=\{\{1\},\{2\},\{3,5,7\},\{4\},\{6\},\{8\}\}$
(f) $\mathcal{P}=\{\{1\},\{2\},\{3,4,5,7\},\{6,8\}\}$
(g) $\mathcal{P}=\{\{1\},\{2\},\{3,4,5,7\},\{6\},\{8\}\}$
(h) $\mathcal{P}=\{\{1,6\},\{2\},\{3,7\},\{4\},\{5\},\{8\}\}$
(i) $\mathcal{P}=\{\{1,6\},\{2\},\{3,5,7\},\{4\},\{8\}\}$
(j) $\mathcal{P}=\{\{1,6,8\},\{2\},\{3,5,7\},\{4\}\}$
(k) $\mathcal{P}=\{\{1,6\},\{2\},\{3,4,7\},\{5\},\{8\}\}$
(l) $\mathcal{P}=\{\{1,6\},\{2\},\{3,4,5,7\},\{8\}\}$
(m) $\mathcal{P}=\{\{1,6,8\},\{2\},\{3,4,5,7\}\}$
(n) $\mathcal{P}=\{\{1,6,8\},\{2,3,4,5,7\}\}$
(o) $\mathcal{P}=\{\{1,2,3,4,5,6,7,8\}\}$

The colorings shown in (a) - (c) are balanced $K$-colorings, hence they define a partial synchronization manifold for the systems with either coupling (2) or coupling (3). Figures $(d)-(n)$ show relaxed balanced $K$-colorings, which define partial synchronization manifolds for coupled systems (1), (3). The trivial relaxed balanced coloring is shown in Figure (o). The latter coloring corresponds to the full synchronization manifold for coupled systems (1), (3).

Consider the coloring shown in Figure 2(a), corresponding to partition $\mathcal{P}=$ $\{\{1\},\{2\},\{3,4,7\},\{5\},\{6\},\{8\}\}$. Let us explain in detail why this coloring is a balanced 6-coloring. We have to verify that each orange node receives the same 


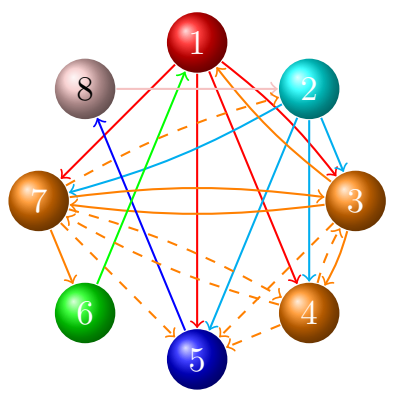

(a) $K=6$

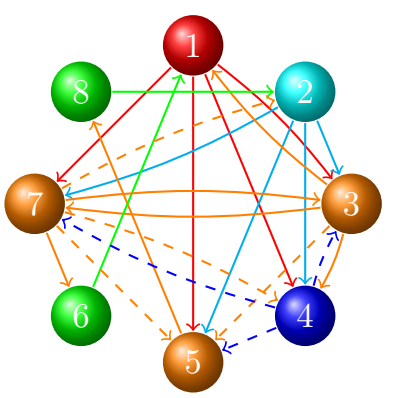

(d) $K=5$

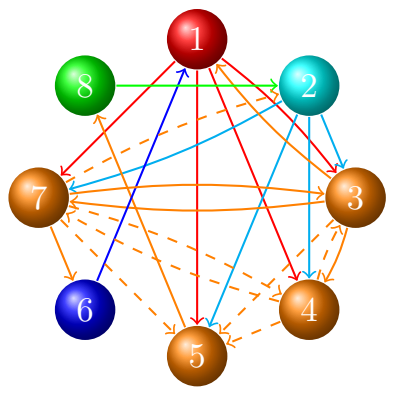

(g) $K=5$

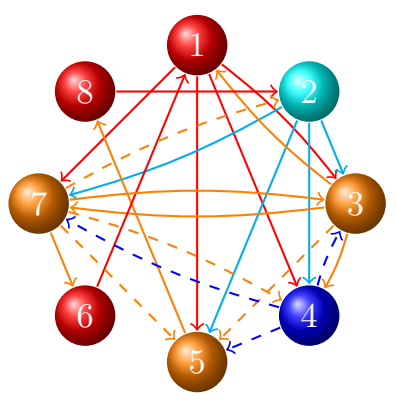

(j) $K=4$

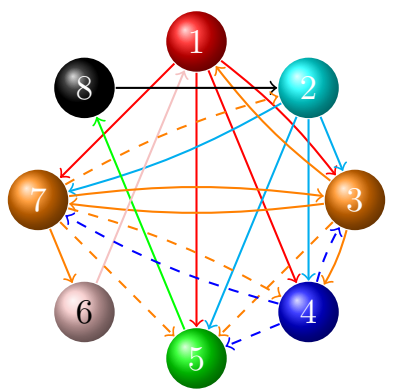

(b) $K=7$

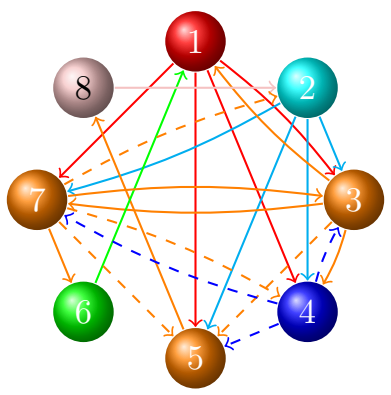

(e) $K=6$

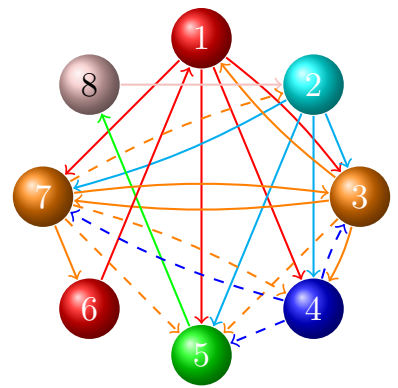

(h) $K=6$

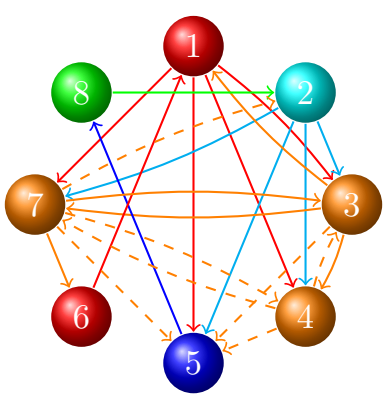

(k) $K=5$

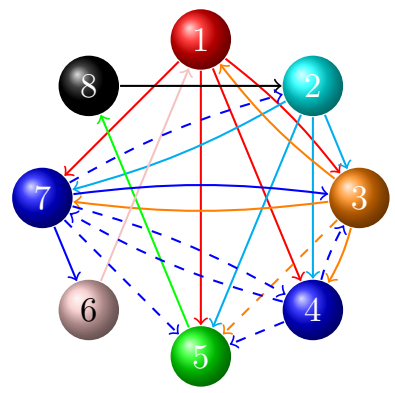

(c) $K=7$

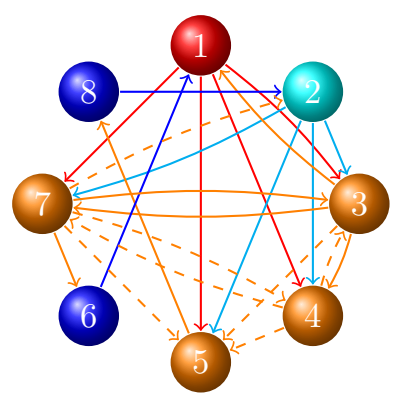

(f) $K=4$

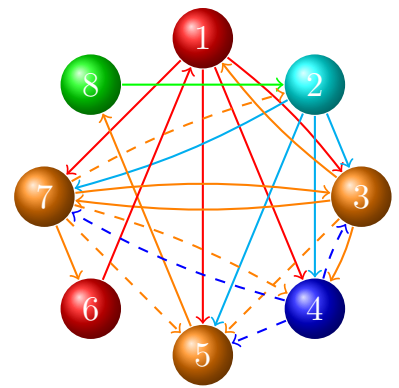

(i) $K=5$

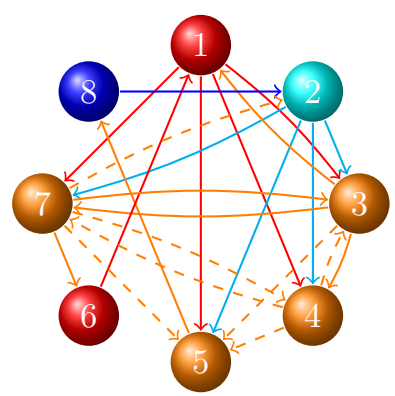

(l) $K=4$

- caption on the next page - 


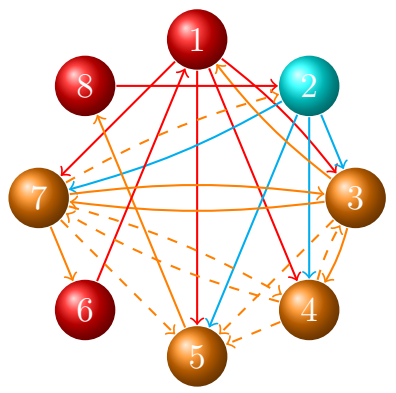

(m) $K=3$

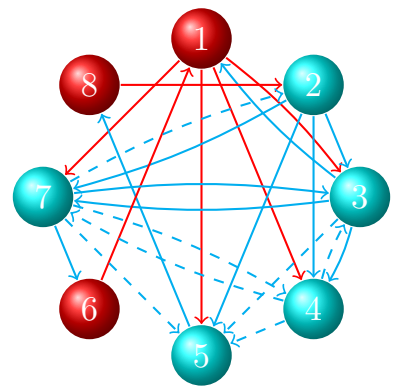

(n) $K=2$

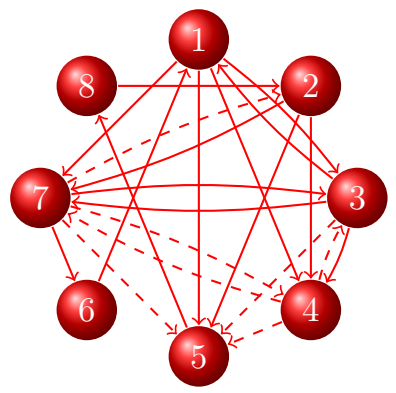

(o) $K=1$

Figure 2. Balanced $K$-coloring (a) - (c), relaxed balanced $K$ colorings (d) - (n), and the trivial relaxed balanced coloring (o) of the example network (case of rationally independent basis weights). Colorings $(\mathrm{d})-(\mathrm{g})$ are balanced $K$-colorings in case $\omega_{1}=2 \omega_{2}$. For illustrative purposes the edges are assigned the same color as the node at their tail.

number of arrows of the same type from nodes with the same color. We see that every orange node receives one solid edge from the red node, one solid edge from the cyan node and one solid edge from an other orange node. In addition, every orange node receives one dashed edge from one other orange node. This shows that the coloring is indeed a balanced coloring for $\mathcal{G}$.

Let us now discuss the relaxed balanced 5-coloring shown in Figure 2(g). We observe that every orange node receives one solid edge from the red node, one solid edge from the cyan node, but the number of solid edges received from other orange nodes differs. A similar situation holds for the dashed edges, hence, we conclude about a relaxed balanced 5-coloring of $\overline{\mathcal{G}}$.

Let us finally consider the case where $\omega_{1}=2 \omega_{2}=2 \omega$ for which $\omega_{1}$ and $\omega_{2}$ are rationally dependent. Hence

$$
A=\omega\left(2 A_{1}+A_{2}\right)=: \omega A^{*} .
$$

Note that this new decomposition is in accordance with Remark 2. Let $\overline{\mathcal{G}}$ be the multi-graph associated with $\mathcal{G}$ and with the decomposition $A=A^{*} \omega$, i.e., such that the number of arrows is defined by the elements of $A^{*}$. The coloring in Figure 3 is a balanced 4-coloring, hence, it defines a partial synchronization manifold for the coupled systems with either coupling (2) or (3). Although this coloring is a balanced 4-coloring of the multi-graph $\overline{\mathcal{G}}$ associated to $A^{*}$ it is not a balanced 4coloring of either $\overline{\mathcal{G}}_{1}$ or $\overline{\mathcal{G}}_{2}$, the multi-graphs associated to $A_{1}$ and $A_{2}$, respectively. For both $\overline{\mathcal{G}}_{1}$ and $\overline{\mathcal{G}}_{2}$ the coloring is however a relaxed balanced 4-coloring, and as such it is (for any $\omega_{1}, \omega_{2}$ ) a relaxed balanced 4-coloring of the whole network. See also Figure 2(f). The same conclusion can be reached for all colorings in subfigures (d) $-(\mathrm{g})$ in Figure 2. Further details are provided in Section 7.

\section{Multiple time-Delays}

Another case where a decomposition of $\mathcal{G}$ is natural occurs when the coupling functions contain multiple time-delays. Let us therefore consider coupling functions 


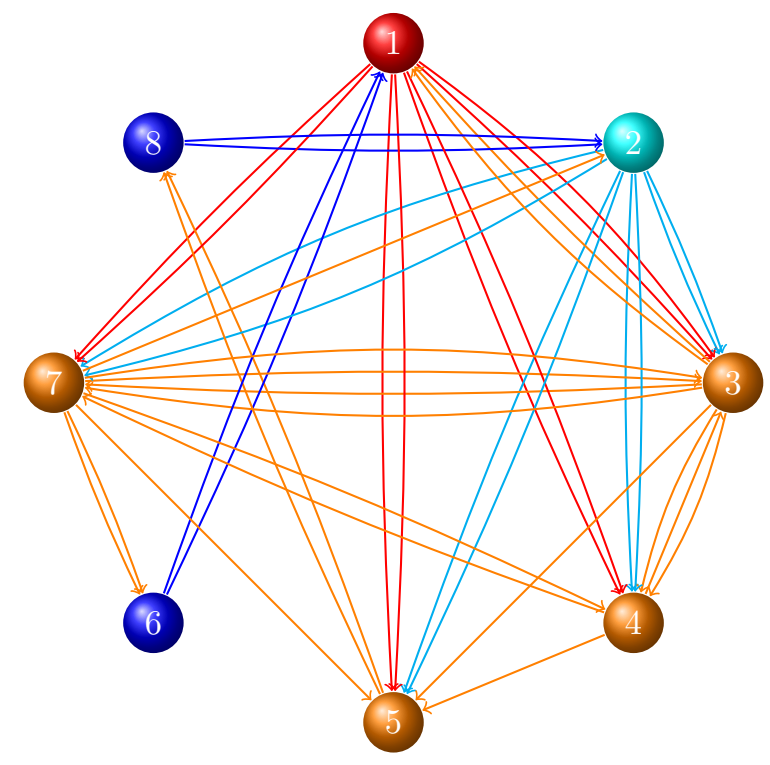

FIGURE 3. A balanced 4-coloring of multi-graph $\overline{\mathcal{G}}$ (corresponding to the case $\omega_{1}=2 \omega_{2}=2 \omega$ ). For illustrative purposes we assigned edges the same color as the node at their tail.

of the form (2) and (3) with multiple time-delays:

$$
u_{i}(t)=\sum_{j \in \mathcal{N}_{i}} a_{i j}\left[y_{j}\left(t-\tau_{i j}\right)-y_{i}(t)\right]
$$

or

$$
u_{i}(t)=\sum_{j \in \mathcal{N}_{i}} a_{i j}\left[y_{j}\left(t-\tau_{i j}\right)-y_{i}\left(t-\tau_{i j}\right)\right],
$$

with $0<\min _{i j}\left(\tau_{i j}\right)<\max _{i j}\left(\tau_{i j}\right)=: \tau$. For both types of coupling functions it is clear that there are at most $N(N-1)$ distinct delays (as there are at most $N(N-1)$ edges in a simple graph with $N$ nodes). Let $p$ be the number of distinct delays such that each delay $\tau_{i j} \in\left\{\tau_{1}, \tau_{2}, \ldots, \tau_{p}\right\}$, where $0<\tau_{1}<\tau_{2}<\ldots<\tau_{p}=\tau$. Then we can write (15) as

$$
u(t)=-\left(D \otimes I_{m}\right) y(t)+\left(\tilde{A}_{1} \otimes I_{m}\right) y\left(t-\tau_{1}\right)+\ldots+\left(\tilde{A}_{p} \otimes I_{m}\right) y\left(t-\tau_{p}\right),
$$

and (16) as

$$
u(t)=-\left(\tilde{L}_{1} \otimes I_{m}\right) y\left(t-\tau_{1}\right)-\ldots-\left(\tilde{L}_{p} \otimes I_{m}\right) y\left(t-\tau_{p}\right),
$$

where $\sum_{\ell=1}^{p} \tilde{A}_{\ell}=A$ and $\tilde{L}_{\ell}=\tilde{D}_{\ell}-\tilde{A}_{\ell}$ with $\tilde{D}_{\ell}=\operatorname{diag}\left(\tilde{A}_{\ell} \mathbf{1}_{N}\right)$. The matrices $\tilde{A}_{\ell}$ are determined as follows:

$$
\tilde{A}_{\ell}=\left(\tilde{a}_{\ell, i j}\right)
$$

with

$$
\tilde{a}_{\ell, i j}= \begin{cases}a_{i j} & \text { if } \tau_{i j}=\tau_{\ell}, \\ 0 & \text { otherwise. }\end{cases}
$$

Accordingly, we can decompose the network $\mathcal{G}$ as follows:

$$
\mathcal{G}(\mathcal{V}, \mathcal{E}, A)=\tilde{\mathcal{G}}_{1}\left(\mathcal{V}, \mathcal{E}_{1}, \tilde{A}_{1}\right) \oplus \ldots \oplus \mathcal{G}_{p}\left(\mathcal{V}, \mathcal{E}_{p}, \tilde{A}_{p}\right) .
$$


Here, $\tilde{\mathcal{E}}_{\ell} \subset \mathcal{V} \times \mathcal{V}$ is the set of edges corresponding to delay $\tau_{\ell}$, i.e., $(i, j) \in \tilde{\mathcal{E}}_{\ell}$ if and only if the $i j^{\text {th }}$ entry of $\tilde{A}_{\ell}$ is nonzero.

Analogously to the results of Sections 3 and 4, we can simplify the analysis of partial synchronization by finding common partial synchronization manifolds for the subnetworks described by $\tilde{\mathcal{G}}_{\ell}, \ell=1, \ldots, p$. The main contribution here is that this simplification does not induce conservatism: the existence criteria for the original networks are equivalent to the existence criteria for all subnetworks.

Theorem 7. Consider the coupled systems (1), (17) on a graph $\mathcal{G}(\mathcal{V}, \mathcal{E}, A)=$ $\tilde{\mathcal{G}}_{1}\left(\mathcal{V}, \mathcal{E}_{1}, \tilde{A}_{1}\right) \oplus \ldots \oplus \mathcal{G}_{p}\left(\mathcal{V}, \mathcal{E}_{p}, \tilde{A}_{p}\right)$. Let $\Pi$ be a permutation matrix of appropriate dimension and associate with $\Pi$ a reordering matrix $R$ such that $R^{\top} \Pi R$ is of the form (6). Then the following statements are equivalent:

1) $\operatorname{ker}\left(I_{N}-\Pi\right)$ is a right invariant subspace of each matrix $\tilde{A}_{\ell}, \ell=1, \ldots, p$;

2) all blocks of each block-structured matrix

$$
R^{\top} \tilde{A}_{\ell} R=\left(\begin{array}{c:c:c:c}
\tilde{A}_{\ell, 11} & \tilde{A}_{\ell, 12} & \ldots & \tilde{A}_{\ell, 1 K} \\
\hdashline \tilde{A}_{\ell, 21} & \tilde{A}_{\ell, 22} & \ldots & \tilde{A}_{\ell, 2 K} \\
\hdashline \vdots & \ddots & \ddots & \vdots \\
\hdashline \tilde{A}_{\ell, K 1} & \tilde{A}_{\ell, K 2} & \ldots & \tilde{A}_{\ell, K K}
\end{array}\right),
$$

with $\tilde{A}_{\ell, i j} \in \mathbb{R}^{k_{i} \times k_{j}}$ and $\ell=1, \ldots, p$, have constant row-sums;

3) there exist $p$ matrices $X_{\tilde{A}_{\ell}}$ such that for each $\ell=1, \ldots, p$,

$$
(I-\Pi) \tilde{A}_{\ell}=X_{\tilde{A}_{\ell}}(I-\Pi) .
$$

Moreover, if the above equivalent conditions are satisfied, then $\mathcal{M}(\Pi)$ is a partial synchronization manifold for the coupled systems (1), (17) on $\mathcal{G}(\mathcal{V}, \mathcal{E}, A)$. If, in addition, the systems (1) are left-invertible, then $\mathcal{M}(\Pi)$ is a partial synchronization manifold for the coupled systems (1), (15) on $\mathcal{G}(\mathcal{V}, \mathcal{E}, A)$ if and only if equivalent conditions 1)-3) hold true.

Theorem 8. Consider the coupled systems (1), (18) on a graph $\mathcal{G}(\mathcal{V}, \mathcal{E}, A)=$ $\tilde{\mathcal{G}}_{1}\left(\mathcal{V}, \mathcal{E}_{1}, \tilde{A}_{1}\right) \oplus \ldots \oplus \mathcal{G}_{p}\left(\mathcal{V}, \mathcal{E}_{p}, \tilde{A}_{p}\right)$. Let $\Pi$ be a permutation matrix of appropriate dimension and associate with $\Pi$ a reordering matrix $R$ such that $R^{\top} \Pi R$ is of the form (6). Then the following statements are equivalent:

1) $\operatorname{ker}\left(I_{N}-\Pi\right)$ is a right invariant subspace of each matrix $\tilde{L}_{\ell}, \ell=1, \ldots, p$;

2) all off-diagonal blocks of each block-structured matrix (19) have constant row-sums;

3) there exist $p$ matrices $X_{\tilde{L}_{\ell}}$ such that for each $\ell=1, \ldots, p$,

$$
(I-\Pi) \tilde{L}_{\ell}=X_{\tilde{L}_{\ell}}(I-\Pi) .
$$

Moreover, if the equivalent conditions above are satisfied, then $\mathcal{M}(\Pi)$ is a partial synchronization manifold for the coupled systems (1), (18) on $\mathcal{G}(\mathcal{V}, \mathcal{E}, A)$. If, in addition, the systems (1) are left-invertible, then $\mathcal{M}(\Pi)$ is a partial synchronization manifold for the coupled systems (1), (18) on $\mathcal{G}(\mathcal{V}, \mathcal{E}, A)$ if and only if equivalent conditions 1)-3) hold true.

Proof. The sufficiency part and equivalence of the conditions in Theorems 7 and 8 essentially follows from Theorem 1 (Proposition 1, Lemmas 1 and 3) and Theorem 2 (Proposition 2, Lemmas 2 and 4), respectively. 
Let us prove the necessary condition for $\mathcal{M}(\Pi)$ to be a partial synchronization manifold for the coupled systems. This proof is by contradiction. Assume that the row-sum condition is not satisfied. Then pick an initial condition on the manifold $\mathcal{M}(\Pi)$ that leads to different inputs to systems in the same cluster. One can first choose $x\left(t_{0}-\tau_{1}\right), \ldots, x\left(t_{0}-\tau_{n}\right)$ that lead to different inputs, and subsequently obtain the full initial condition (a function on the interval $\left[t_{0}-\tau, t_{0}\right]$ ) by interpolation. Then by left-invertibility the solution of the coupled systems can not stay on $\mathcal{M}(\Pi)$ for $t \geq t_{0}$.

Remark 5. Theorems 7-8 are based on a decomposition of the graph according to the different delays. Based on the rational dependency structure of the weighs a further decomposition as in Section 5 is possible. A connection with a balanced coloring can then be made by defining one multi-graph where the definition and type of edges (equivalence relation) are according to the combination (delay value, base weight).

\section{AN ALGORITHM FOR DETECTION OF PARTIAL SYNCHRONIZATION MANIFOLDS}

The characterizations of partial synchronization manifolds, presented in Sections 3-6, correspond to analysis conditions: given a manifold (or a partition of the systems), conditions are established to check whether it corresponds to a partial synchronization manifold. In this section we present an algorithm for the synthesis problem of determining all possible partitions for which the conditions in Lemma 1, respectively Lemma 2, are satisfied for a given graph $\mathcal{G}$.

The detection algorithm, which we discuss in what follows, has been implemented in MATLAB and is available from

http://twr.cs.kuleuven.be/research/software/delay-control/manifolds/

7.1. Description of the algorithm. The algorithm follows the same steps as the one in [20]. It consist of two ingredients, viz. the generation possible partitions and checking the row-sum criteria of the previous sections. The latter takes into account the type of delay-coupling and, possibly, the dependency structure of the weighs. For an algorithm for finding partial synchronization manifolds based on the eigenvalue decomposition of $A$ and invariant subspaces, we refer to [1].

To make the paper self contained we briefly discuss the generation of all partitions. This is done by induction on the number of systems $k$ and graphically explained in Table 1. System 1 always gets code 0 . If there are two systems, then there are two possible partitions: one group, coded by 00, or two groups, coded by 01 . Suppose now that we add a third system. For every possible partition of Systems 1 and 2, we can generate partitions for Systems 1-3. Starting from partition 00 we can either add System 3 to the group or put it into a new group, coded by 000 and 001, respectively. Similarly, starting from partition 01, we have three possibilities: we can add System 3 to System 1 (code 010), add it to System 2 (code 011) or put into a new group (012), etc.

Recall that a partition that corresponds to a partial synchronization manifold is called a viable partition. To check whether a partition is a viable partition, the algorithm performs the row-sum test, corresponding to Lemma 1 or Lemma 2, depending on the coupling type, (2) or (3). If all elements of $A$ are integers or if an explicit decomposition of $A$ as in (12) is known (i.e., all weights are integer combinations of rationally independent base weights), then, by Theorems 5 and 6 , 


\begin{tabular}{|r|r|r|r|r|}
\hline System & Systems & Systems & Systems & $\cdots$ \\
1 & 1,2 & $1,2,3$ & $1,2,3,4$ & \\
\hline \hline 0 & 00 & 000 & 0000 & $\cdots$ \\
& & & 0001 & \\
& & 001 & 0010 & \\
& & & 0011 & \\
& & & 0012 & \\
& & & & $\ddots$ \\
& & 01 & 0100 & \\
& & & 0101 & \\
& & 0102 & \\
& & & 0110 & \\
& & & 0111 & \\
& & 012 & 0120 & \\
& & & 0121 & \\
& & & 0122 & \\
& & & & \\
& & & & $\ddots$ \\
\hline
\end{tabular}

TABLE 1. Generating the set of all partitions of a number of systems.

only row-sum tests on matrices with integer elements need to be made. Hence, the detection algorithm can be carried out in exact arithmetic, without making conditions more stringent. In this case matrices $A_{\ell}$ are represented by a structure passed as input argument. In the other case, the row-sum test of Lemma 1 or Lemma 2 need to be performed in finite precision arithmetic, where the user can specify the tolerance on the difference between computed row-sums for accepting the partition.

For each type of coupling the algorithm detects all partitions for which the corresponding row-sum criteria holds true. In case of an explicit decomposition of $A$ as in (12) the algorithm performs the row-sum test on each matrix $A_{\ell}$, which may be advantageous as the row-sum tests on matrices $A_{\ell}$ can be done in exact arithmetic. Recall that, if the basis weights $\omega_{\ell}$ are rationally independent, performing the row-sum test on all matrices $A_{\ell}$ does not introduce any conservatism. In case the basis weights are rationally dependent, there are two possibilities. The first possibility corresponds to performing the row-sum tests on matrix $A$ (with, possibly, non-integer entries) for identifying all viable partitions (in this case the input to the algorithm is matrix $A$ ). The second possibility consists of rewriting the decomposition in accordance to Remark 2 , and using $\left(A_{1}^{*}, \ldots, A_{p}^{*}\right)$ as input.

Under the additional hypothesis that the systems are left-invertible, the algorithm returns all possible partial synchronization manifolds for a given network. The price to pay is that, due to the combinatorial nature of the algorithm (the number of possible partitions of $N$ systems, known as the $N$-th Bell number, exhibits an exponential like growth as a function of $N$ ), it scales badly with the network size. By relaxing requirements to computing selected partitions /clusters 
(instead of all) or to computing approximate invariant manifolds, algorithms applicable to large networks can be obtained. See also [3, 4, 20] for algorithms to compute the minimal balanced coloring of a graph in the delay free case. However, this is beyond the scope of this paper.

7.2. Example. We continue with the example introduced in Sections 3.5 and 5.2, and illustrate the use of the implementation in MATLAB. Let us first consider the case where $\omega_{1}$ and $\omega_{2}$ are rationally independent, and let matrices $A_{1}$ and $A_{2}$ be as in Section 5.2.

The partitions corresponding to partial synchronization manifolds for the case of coupling (2) can be found using the following commands:

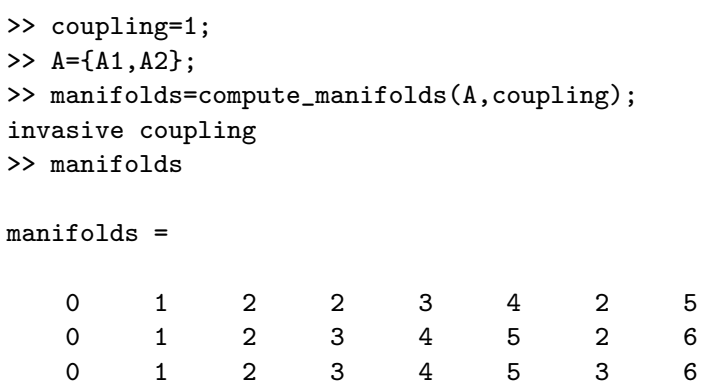

$\begin{array}{llllllll}0 & 1 & 2 & 2 & 3 & 4 & 2 & 5 \\ 0 & 1 & 2 & 3 & 4 & 5 & 2 & 6 \\ 0 & 1 & 2 & 3 & 4 & 5 & 3 & 6\end{array}$

The first row of the output of the algorithm corresponds to the partition shown in Figure 2(a), the second row corresponds to the partition shown in Figure 2(b), and the third row corresponds to the partition shown in Figure 2(c). Note that all row-sum tests are carried out in exact arithmetic as the elements of $A$ are integers.

In case of coupling type (3) we get:

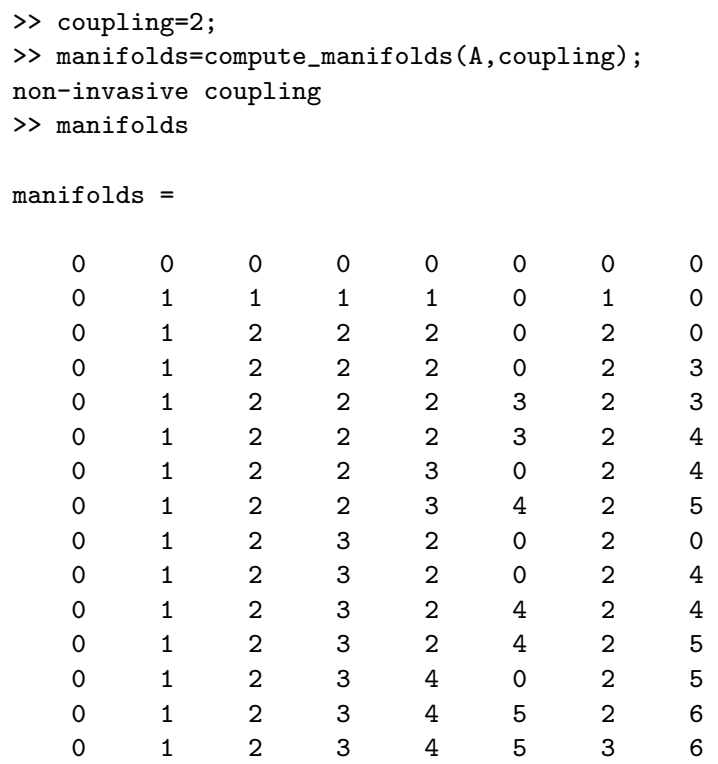

The first row of the output is the trivial partition, which corresponds to one cluster that contains all systems, i.e. the full synchronization manifold, which 
always exists for coupling (3). For this type of coupling the algorithm detected twelve partitions additional to the three detected for coupling (2) (obviously, all valid partitions for coupling (2) are valid partitions for coupling (3)). Given the small number of systems and that, at a first glance, the graph in Figure 1 is not very symmetrical, it is quite interesting that fifteen partial synchronization manifolds are present. All viable partitions found by the algorithm are graphically presented in Figure 2.

Let us subsequently consider the case where $\omega_{1}=2 \omega_{2}=2 \omega$, for which the trivial decomposition

$$
A=\left(2 A_{1}+A_{2}\right) \omega=A^{*} \omega
$$

holds. Note that matrix $A^{*}$ has integer entries. Executing the algorithm for coupling (2) with integer matrix $A^{*}$ as input gives the following result:

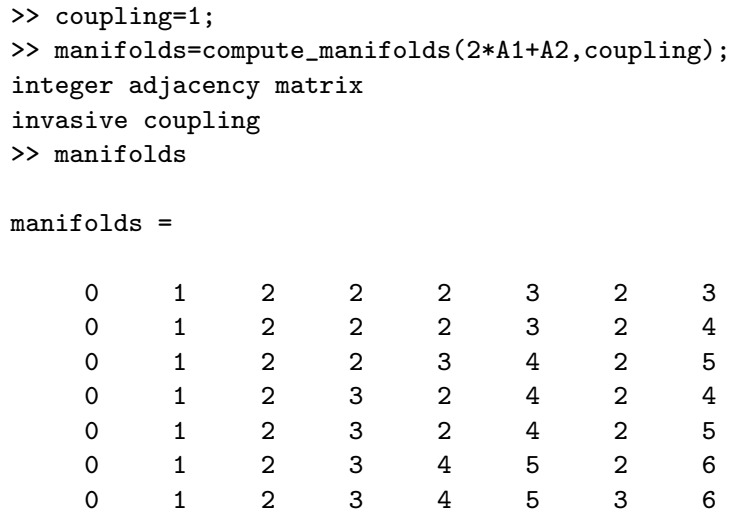

$\begin{array}{llllllll}0 & 1 & 2 & 2 & 2 & 3 & 2 & 3 \\ 0 & 1 & 2 & 2 & 2 & 3 & 2 & 4 \\ 0 & 1 & 2 & 2 & 3 & 4 & 2 & 5 \\ 0 & 1 & 2 & 3 & 2 & 4 & 2 & 4 \\ 0 & 1 & 2 & 3 & 2 & 4 & 2 & 5 \\ 0 & 1 & 2 & 3 & 4 & 5 & 2 & 6 \\ 0 & 1 & 2 & 3 & 4 & 5 & 3 & 6\end{array}$

We observe that in this case we have four additional viable partitions compared to the case of general, rationally independent $\omega_{1}$ and $\omega_{2}$. These additional viable partitions are graphically represented in Figures $2(\mathrm{~d})-2(\mathrm{~g})$. For coupling functions (3) no additional viable partitions exist.

Let us finally assume a network with adjacency matrix

$$
A^{*}=\left(\begin{array}{cccccccc}
0 & 0 & 1.9968 & 0 & 0 & 1.9976 & 0 & 0 \\
0 & 0 & 0 & 0 & 0 & 0 & 1.0254 & 1.9892 \\
1.9989 & 1.9922 & 0 & 0.9933 & 0 & 0 & 1.9868 & 0 \\
1.9844 & 1.9894 & 2.0016 & 0 & 0 & 0 & 1.0013 & 0 \\
2.0192 & 1.9823 & 0.9972 & 0.9922 & 0 & 0 & 0.9856 & 0 \\
0 & 0 & 0 & 0 & 0 & 0 & 2.0130 & 0 \\
1.9935 & 1.9895 & 1.9885 & 1.0055 & 0 & 0 & 0 & 0 \\
0 & 0 & 0 & 0 & 2.0091 & 0 & 0 & 0
\end{array}\right) .
$$

This adjacency matrix is actually the matrix $2 A_{1}+A_{2}$ with the non-zero elements contaminated with some noise. One may think of this matrix $A^{*}$ as the adjacency matrix obtained using experiments done on the "true network" with adjacency matrix $2 A_{1}+A_{2}$. As the entries of $A^{*}$ are non-integer, we should run the algorithm with a pre-specified tolerance. The following few lines of code illustrate how the identified viable partitions depend on the pre-specified tolerance.

> coupling=1; 


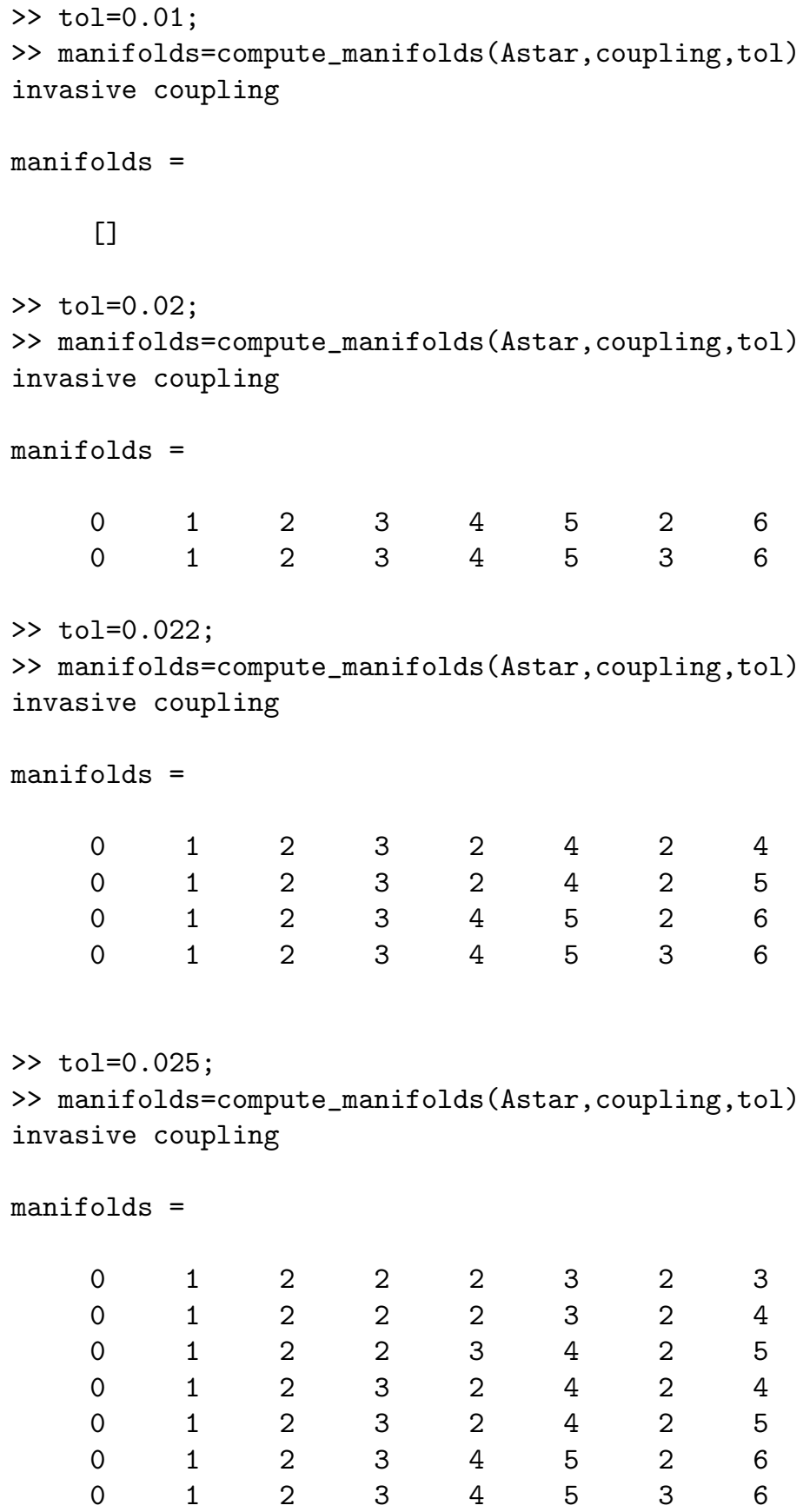

$\begin{array}{llllllll}0 & 1 & 2 & 3 & 4 & 5 & 3 & 6\end{array}$

$>$ tol $=0.025$;

$\begin{array}{llllllll}0 & 1 & 2 & 2 & 2 & 3 & 2 & 3 \\ 0 & 1 & 2 & 2 & 2 & 3 & 2 & 4 \\ 0 & 1 & 2 & 2 & 3 & 4 & 2 & 5 \\ 0 & 1 & 2 & 3 & 2 & 4 & 2 & 4 \\ 0 & 1 & 2 & 3 & 2 & 4 & 2 & 5 \\ 0 & 1 & 2 & 3 & 4 & 5 & 2 & 6 \\ 0 & 1 & 2 & 3 & 4 & 5 & 3 & 6\end{array}$

We observe that the number of viable partitions detected by the algorithm depends on the pre-specified tolerance. We need to set the tolerance to 0.025 in order to detect the viable partitions of the "true" network with adjacency matrix $2 A_{1}+A_{2}$. The algorithm does not return additional viable partitions as the tolerance is increased up to acceptable levels. Of course, if the tolerance is set to a value 
close to the smallest element in $A^{*}$, which is about 1 in this case, the algorithm may return additional partitions. However, these additional partitions found for large value of the tolerance are unlikely to be viable partitions.

\section{CONCLUding REMARKS}

We have presented a number of equivalent conditions for the existence of partial synchronization manifolds $\mathcal{M}(\Pi)$ in networks of systems (1) that interact via invasive time-delay coupling (2) or non-invasive time-delay coupling (3). We have shown that these conditions are necessary and sufficient under the mild condition that system (1) is left-invertible.

Particular attention has been paid to decomposable networks. As a central contribution, it has been shown that, in two cases, it suffices to find common partial synchronization manifolds for the subnetworks, without introducing conservatism. These cases correspond to a decomposition based on the dependency structure of the weights, with rationally independent basis weights, and according to different delay values. It has also been shown with a counterexample that when the basis weighs are rationally dependent, resorting to finding common partial synchronization manifolds for the subnetworks may be too restrictive, i.e., one might not be able to detect all manifolds.

We also made connections with (generalization of) the concept of balanced coloring of multi-graphs, and presented an algorithm for determining partial synchronization manifolds.

\section{ACKNOWLEDGEMENTS}

This research was supported by the Programme of Interuniversity Attraction Poles of the Belgian Federal Science Policy Office (IAP P6- DYSCO), by OPTEC, the Optimization in Engineering Center of the KU Leuven and by the project G.0712.11N of the Research Foundation-Flanders (FWO).

Cees van Leeuwen is supported by an Odysseus grant from the Research FoundationFlanders (FWO).

\section{REFERENCES}

[1] A.D. Aguiar and A.P.S. Dias. The lattice of synchrony subspaces of a coupled cell network: characterization and computation algorithm. J. Nonlinear Sci., 24:949-996, 2014.

[2] A.D. Aguiar, A.P.S. Dias, M. Golubitsky, M. da Conceição, and A. Leite. Bifurcations from regular quotient networks: A first insight. Physica D, 238:137-155, 2009.

[3] J. W. Aldis. A polynomial time algorithm to determine maximal balanced equivalence relations. Int. J. Bifurcation and Chaos, 18:407-427, 2008.

[4] I. Belykh and M. Hasler. Mesoscale and clusters of synchrony in networks of bursting neurons. Chaos, 21(016106), 2011.

[5] V. N. Belykh, I. V. Belykh, and M. Hasler. Hierarchy and stability of partially synchronous oscillations of diffusively coupled dynamical systems. Phys. Rev. E, 62(5):6332-6345, 2000.

[6] M. V. L. Bennet and R. S. Zukin. Electrical coupling and neuronal synchronization in the mammalian brain. Neuron, 41:495-511, 2004.

[7] B. Bollobás. Modern graph theory, volume 184 of Graduate Texts in Mathematics. SpringerVerlag, 1998.

[8] C. I. Byrnes, A. Isidori, and J. C.Willems. Passivity, feedback equivalence, and the global stabilization of minimum phase nonlinear systems. IEEE trans. Auto. Contr., 36(11):1228$1240,1991$.

[9] S.-J. Chung and J.-J. E. Slotine. Coorperative robot control and concurrent synchronization of Lagrangian systems. IEEE trans. Robotics, 25(3):686-700, 2009. 
[10] J. J. Collins and I. N. Stewart. Coupled nonlinear oscillators and the symmetries of animal gaits. J. Nonlinear Sci., 3:349-392, 1993.

[11] S. Coombes. Neuronal networks with gap junctions: A study of piecewise linear planar neuron models. SIAM J. Appl. Dyn. Syst., 7(3):1101-1129, 2008.

[12] K. M. Cuomo, A. V. Oppenheim, and S. H. Strogatz. Synchronization of Lorenz based chaotic circuits with applications to communications. IEEE trans. Circ. Syst. II, 40(10):626-633, 1993.

[13] B. Dionne, M. Golubitsky, and I. Stewart. Coupled cells with internal symmetry: I. wreath products. Nonlinearity, 9(2):559-574, 1996.

[14] B. Dionne, M. Golubitsky, and I. Stewart. Coupled cells with internal symmetry: II. direct products. Nonlinearity, 9(2):575-599, 1996.

[15] M. Golubitsky, I. Stewart, and A. Török. Patterns of synchrony in coupled cell networks with multiple arrows. SIAM J. Appl. Dynam. Sys., 4(1):78-100, 2005.

[16] C. M. Gray. Synchronous oscillations in neuronal systems: Mechanisms and functions. $J$. Comp. Neuroscience, 1(1-2):11-38, 1994.

[17] Nijmeijer H and A. J. v.d. Schaft. Nonlinear Dynamical Control Systems. Springer-Verlag, New York, 1990.

[18] R. M. Hirschorn. Invertibility of multivariable nonlinear control systems,. IEEE Trans. Aut. Contr., 24:855-865, 1979.

[19] K. Judd. Networked dynamical systems with linear coupling: Synchronizaiton patterns, coherence and other behaviors. Chaos, 23(043112), 2013.

[20] H. Kamei and P. J. A. Cock. Computation of balanced equivalence relations and their lattice for a coupled cell network. SIAM J. Appl. Dyn. Syst., 12(1):352-382, 2013.

[21] K. Kaneko. Relevance of dynamic clustering to biological networks. Physica D, 75(1-3):55-73, 1994.

[22] J. G. Mancilla, T. J. Lewis, D. J. Pinto, J. Rinzel, and B. W. Connors. Synchronization of electrically coupled pairs of inhibitory interneurons in neocortex. J. Neurosci., 27:2058-2073, 2007.

[23] W. Michiels and S.-I. Niculescu. Stability, control, and computation for time-delay systems. An eigenvalue based approach. SIAM, 2014.

[24] V. Nicosia, M. Valencia, M. Chavez, A. Díaz-Guilera, and V. Latora. Remote synchronization reveals network symmetries and functional modules. Phys. Rev. Lett., 110(174102), 2013.

[25] H. Nijmeijer. Invertibility of affine nonlinear control systems: A geometric approach. Syst. Contr. Lett., 2(3):163-168, 1982.

[26] H. Nijmeijer and A. Rodriguez-Angeles. Synchronization of mechanical systems. World Scientific, Singapore, 2003.

[27] G. Orosz. Decomposition of nonlinear delayed networks around cluster states with applications to neurodynamics. SIAM J. of Appl. Dyn. Syst., 13(4):1353-1386, 2014.

[28] L. M. Pecora, F. Sorrentino, A. M. Hagerstrom, T. E. Murphy, and R. Roy. Cluster synchronization and isolated desynchronization in complex networks with symmetries. Nature comm., 5(4079), 2014.

[29] M. Perez-Armendariz, C. Roy, D. C. Spray, and M. V. L. Bennett. Biophysical properties of gap juntions between freshly dispersed pairs of mouse pancreatic beta cells. Biophys. J., 59:76-92, 1991.

[30] Arkady Pikovsky, Micheal Rosenblum, and Jürgen Kurths. Synchronization. Cambridge University Press, 2 edition, 2003.

[31] A. Pogromsky, T. Glad, and H. Nijmeijer. On diffusion driven oscillations in coupled dynamical systems. Int. J. Bif. Chaos, 9(4):629-644, 1999.

[32] A. Pogromsky, G. Santoboni, and H. Nijmeijer. Partial synchronization: from symmetry towards stability. Physica D, 172(1-4):65-87, 2002.

[33] A. Y. Pogromsky. A partial synchronization theorem. Chaos, 18:037107, 2008. Erratum: Chaos 19: 049901, 2009.

[34] A. Rodriguez-Angeles and H. Nijmeijer. Coordination of two robot manipulators based on position measurements only. Int. J. Control, 74(13), 2001.

[35] A. Sherman, J. Rinzel, and J. Keizer. Emergence of organized bursting in clusters of pancreatic beta-cells by channel sharing. Biophys. J., 54(3):411-425, 1998.

[36] R. Sipahi, S. Niculescu, C. T. Abdallah, W. Michiels, and K. Gu. Stability and stabilization of systems with time delay. IEEE Contr. Syst., 31(1):38-65, 2011. 
[37] E. Steur, T. Oguchi, C. v. Leeuwen, and H. Nijmeijer. Partial synchronization in diffusively time-delay coupled oscillator networks. Chaos, 22(043144), 2012.

[38] E. Steur, C. van Leeuwen, and W. Michiels. Partial synchronization manifolds for linearly time-delayed coupled systems. In 21th International Symposium on Mathematical Theory of Networks and Systems (MTNS 2014), Groningen, the Netherlands, 2014.

[39] I. Stewart, M. Golubitsky, and M. Pivato. Symmetry groupoids and patterns of synchrony in coupled cell networks. SIAM J. Appl. Dyn. Syst., 2(4):609-646, 2003.

[40] S. H. Strogatz. Sync: the emerging science of spontaneous order. Hyperion, 1 edition, 2003.

[41] P. J. Uhlhaas and W. Singer. Neural synchrony in brain disorders: Relevance for cognitive dysfunctions and pathophysiology. Neuron, 52(1):155-168, 2006.

[42] H. U. Ünal and W. Michiels. Prediction of partial synchronization in delay-coupled nonlinear oscillators, with application to Hindmarsh-Rose neurons. Nonlinearity, 26(12):3101-3126, 2013.

[43] C. W. Wu and L. O. Chua. Synchronization in an array of linearly coupled dynamical systems. IEEE trans. Circ. Syst. I, 42(8):430-447, 1995.

[44] M. Zanin, J. M. Buldú, and S. Boccaletti. Networks of springs: A practical approach. Int. J. Bif. Chaos, 20(3):937-942, 2010.

Laboratory for Perceptual Dynamics, Faculty of Psychology and Educational Sciences, KU Leuven, Tiensestraat 102, Box 3711, Leuven,B-3000, Belgium

Current address: Department of Mechanical Engineering and Institute for Complex and Molecular Systems, Eindhoven University of Technology, P.O. Box. 513, 5600 MB, Eindhoven, the Netherlands

E-mail address: e.steur@tue.nl

Department of Electrical and Electronics Engineering, Anadolu University, EskiseHIR 26555, TURKEY

E-mail address: huunal@anadolu.edu.tr

Laboratory for Perceptual Dynamics, Faculty of Psychology and Educational Sciences, KU Leuven, Tiensestraat 102, Box 3711, Leuven,B-3000, Belgium

E-mail address: cees.vanleeuwen@ppw.kuleuven.be

Department of Computer Science, Ku Leuven, Heverlee 3001, Belgium

E-mail address: wim.michiels@cs.kuleuven.be 